\title{
QCD SUM RULES DESCRIPTION OF NUCLEONS IN ASYMMETRIC NUCLEAR MATTER
}

\author{
E.G. Drukarev, M.G. Ryskin, V.A. Sadovnikova \\ Petersburg Nuclear Physics Institute, \\ Gatchina, St. Petersburg 188300, Russia
}

\begin{abstract}
We calculate the nucleon parameters in isospin asymmetric nuclear matter using the QCD sum rules. The nucleon self-energies $\Sigma_{v}$ and $\Sigma_{s}^{*}$ are expressed in terms of the in-medium values of QCD condensates. The simple approximate expressions for the self-energies are obtained in terms of these condensates. Relation between successive inclusion of the condensates and the meson-exchange picture of the nucleon interaction with medium is analyzed. The values of the self-energies and of the symmetry energy agree with those obtained by the methods of nuclear physics.
\end{abstract}

\section{INTRODUCTION}

In this paper we investigate the vector and scalar self-energies of nucleons in nuclear matter composed by the neutrons and protons, distributed with densities $\rho_{n}$ and $\rho_{p}$. We calculate the dependence on the total density

$$
\rho=\rho_{p}+\rho_{n}
$$

and on the asymmetry parameter

$$
\beta=\frac{\rho_{n}-\rho_{p}}{\rho_{p}+\rho_{n}}
$$

(another conventional presentation of the asymmetry parameter is $\beta=\frac{N-Z}{N+Z}=1-\frac{2 Z}{A}$ with $N$ and $Z$ standing for the total number of neutrons and protons, while $A=N+Z$ ). We present also the equations for the single-particle potential energies. The results are obtained by QCD sum rules approach.

The QCD sum rules were invented by Shifman et al. [1] to express the hadron parameters through the vacuum expectation values of $\mathrm{QCD}$ operators. Being initially used for the mesons, the method was expanded by Ioffe [2, 3] to the description of the baryons. The approach succeeded in describing the static characteristics as well as some of the dynamical characteristics of the hadrons in vacuum - see, e.g., the reviews [4, 5]. 
The main idea is to consider the correlation function

$$
\Pi_{0}(q)=q_{\mu} \gamma^{\mu} \Pi_{0}^{q}\left(q^{2}\right)+I \Pi_{0}^{I}\left(q^{2}\right)
$$

( $I$ stands for the unit $4 \times 4$ matrix) describing the propagation of the system with the quantum numbers of the hadron, in the different regions of values of the momentum $q^{2}$, where certain information on its behavior is available. The asymptotic freedom of QCD enables to present $\Pi_{0}^{j}\left(q^{2}\right)(j=q, I)$ at $q^{2} \rightarrow-\infty$ as the power series of $q^{-2}$ and QCD coupling $\alpha_{s}$. On the other hand, the imaginary part of $\Pi_{0}\left(q^{2}\right)$ at $q^{2}>0$ can be described in terms of the observable hadrons. This prompts to consider the dispersion relation for the function $\Pi_{0}^{j}\left(q^{2}\right)[1]$

$$
\Pi_{0}\left(q^{2}\right)=\frac{1}{\pi} \int \frac{\operatorname{Im} \Pi_{0}\left(k^{2}\right)}{k^{2}-q^{2}} d k^{2}
$$

at $q^{2} \rightarrow-\infty$. The coefficients of the expansion of the left-hand side (lhs) of the functions $\Pi_{0}^{j}\left(q^{2}\right)$ in powers of $q^{-2}$ are the expectation values of the local operators constructed of the quark and gluon fields, which are called "condensates". Such presentation, known as the operator product expansion (OPE) 6] provides the perturbative expansion of the shortdistance effects, while the nonperturbative physics is contained in the condensates. The usual treatment of the right-hand side (rhs) of Eq. (4) consists in "pole + continuum" presentation, in which the lowest laying pole is singled out while the higher states are approximated by the continuum described by the pure perturbative OPE contribution. Thus, Eq. (4) ties the values of QCD condensates with the characteristics of the lowest hadronic state. Such interpretation requires that the contribution of the pole to the rhs of Eq. (4) exceeds the contribution of the continuum.

The OPE of the lhs of Eq. (4) becomes increasingly true, while the value of $\left|q^{2}\right|$ increases. On the other hand, the "pole + continuum" model becomes more accurate while $\left|q^{2}\right|$ decreases. The important assumption is that the two presentations are close in certain intermediate region of the values of $q^{2}$. To improve the overlap of the QCD and the phenomenological descriptions, one usually applies certain mathematical anzats, i.e., the Borel transform. The Borel transformed dispersion relations (44) are known as QCD sum rules [1, 2].

For example, the QCD sum rules for the nucleon provided a connection between the nucleon mass and the scalar quark condensate $\langle 0|\bar{q} q| 0\rangle$ [2, 3]. A more detailed analysis requires inclusion of the expectation values of the higher dimension, e.g., the gluon condensate $\left\langle 0\left|\frac{\alpha_{s}}{\pi} G_{\mu \nu} G_{\mu}^{\nu}\right| 0\right\rangle$ and the four-quark condensate $\langle 0|\bar{q} q \bar{q} q| 0\rangle$. Two other unknowns of nucleon QCD sum rules equations are the residue of the nucleon pole $\lambda_{0}^{2}$ and the effective continuum threshold $W_{0}^{2}$.

Later the QCD sum rules were applied for investigation of modified nucleon parameters in the symmetric nuclear matter [7, 8. They were based on the Borel-transformed dispersion relation for the function $\Pi_{m}(q)$ describing the propagation of the system with the quantum numbers of the nucleon (the proton) in the nuclear matter. Considering nuclear matter as a 
system of $A$ nucleons with momenta $p_{i}$, one introduces the vector

$$
p=\frac{\Sigma p_{i}}{A},
$$

which is thus $p \approx(m, 0)$ in the rest frame of the matter. The function $\Pi_{m}(q)$ can be presented as $\Pi_{m}(q)=\Pi_{m}\left(q^{2}, \varphi(p, q)\right)$ with the arbitrary function $\varphi(p, q)$ being kept constant in the dispersion relations in $q^{2}$.

The general form of the function $\Pi_{m}$ can thus be presented as

$$
\Pi_{m}(q)=q_{\mu} \gamma^{\mu} \Pi_{m}^{q}\left(q^{2}, s\right)+p_{\mu} \gamma^{\mu} \Pi_{m}^{p}\left(q^{2}, s\right)+I \Pi_{m}^{I}\left(q^{2}, s\right) .
$$

The in-medium QCD sum rules are the Borel-transformed dispersion relations for the components $\Pi_{m}^{j}\left(q^{2}, s\right)(j=q, p, I)$

$$
\Pi_{m}^{j}\left(q^{2}, s\right)=\frac{1}{\pi} \int \frac{\operatorname{Im} \Pi_{m}^{j}\left(k^{2}, s\right)}{k^{2}-q^{2}} d k^{2} .
$$

The spectrum of the function $\Pi_{m}(q)$ is much more complicated than that of the function $\Pi_{0}(q)$. The choice of the function $\varphi(p, q)$ is dictated by the attempts to separate the singularities connected with the nucleon in the matter from those connected with the properties of the matter itself. Since the latter manifest themselves as singularities in the variable $s=(p+q)^{2}$, the separation can be done by putting $\varphi(p, q)=(p+q)^{2}$ and by fixing [7, 8, , 9]

$$
\varphi(p, q)=(p+q)^{2} \equiv s=4 E_{0 F}^{2}
$$

with $E_{0 F}$ being the total nucleon energy at the Fermi surface.

By using Eq. (77) the characteristics of the nucleon in nuclear matter can be expressed through the in-medium values of QCD condensate. The possibility of extension of "pole + continuum" model [1, 2] to the case of finite densities was shown in [7]-[10].

The lowest order of OPE of the lhs of Eq. (7) can be presented in terms of the vector and scalar condensates [7]-[10]. Vector condensates $v_{\mu}^{i}=\left\langle M\left|\bar{q}^{i} \gamma_{\mu} q^{i}\right| M\right\rangle$ of the quarks with the flavor $i(|M\rangle$ denotes the ground state of the matter) are the linear functions of the nucleon densities $\rho_{n}$ and $\rho_{p}$. In the asymmetric matter both $\mathrm{SU}(2)$ symmetric and asymmetric condensates

$$
v_{\mu}=\left\langle M\left|\bar{u}(0) \gamma_{\mu} u(0)+\bar{d}(0) \gamma_{\mu} d(0)\right| M\right\rangle=v_{\mu}^{u}+v_{\mu}^{d}
$$

and

$$
v_{\mu}^{(-)}=\left\langle M\left|\bar{u}(0) \gamma_{\mu} u(0)-\bar{d}(0) \gamma_{\mu} d(0)\right| M\right\rangle=v_{\mu}^{u}-v_{\mu}^{d}
$$

obtain nonzero values. In the rest frame of the matter $v_{\mu}^{i}=v^{i} \delta_{\mu 0}, v_{\mu}=v \delta_{\mu 0}, v_{\mu}^{(-)}=v^{(-)} \delta_{\mu 0}$. We can present

$$
v^{i}=\left\langle n\left|\bar{q}^{i} \gamma_{0} q^{i}\right| n\right\rangle \rho_{n}+\left\langle p\left|\bar{q}^{i} \gamma_{0} q^{i}\right| p\right\rangle \rho_{p} .
$$

The values $\left\langle N\left|\bar{q}^{i} \gamma_{0} q^{i}\right| N\right\rangle$ are just the numbers of the valence quarks in the nucleons $\left\langle n\left|\bar{u} \gamma_{0} u\right| n\right\rangle=$ $\left\langle p\left|\bar{d} \gamma_{0} d\right| p\right\rangle=1,\left\langle p\left|\bar{u} \gamma_{0} u\right| p\right\rangle=\left\langle n\left|\bar{d} \gamma_{0} d\right| n\right\rangle=2$, and thus

$$
v^{u}=\rho_{n}+2 \rho_{p}=\rho\left(\frac{3}{2}-\frac{\beta}{2}\right), \quad v^{d}=2 \rho_{n}+\rho_{p}=\rho\left(\frac{3}{2}+\frac{\beta}{2}\right) .
$$


Hence, we obtain

$$
v(\rho)=v_{N} \rho, \quad v^{(-)}(\rho, \beta)=\beta v_{N}^{(-)} \rho
$$

with

$$
v_{N}=3, \quad v_{N}^{(-)}=-1 .
$$

The lhs of Eq. (17) contains the SU(2) symmetric scalar condensate

$$
\kappa_{m}(\rho)=\langle M|\bar{u}(0) u(0)+\bar{d}(0) d(0)| M\rangle,
$$

and $\mathrm{SU}(2)$ asymmetric one

$$
\zeta_{m}(\rho, \beta)=\langle M|\bar{u}(0) u(0)-\bar{d}(0) d(0)| M\rangle .
$$

These condensates can be presented as

$$
\kappa_{m}(\rho)=\kappa_{0}+\kappa(\rho),
$$

$\kappa_{0}=\kappa_{m}(0)$ is the vacuum value,

$$
\kappa(\rho)=\kappa_{N} \rho+\ldots, \quad \kappa_{N}=\langle N|\bar{u} u+\bar{d} d| N\rangle,
$$

and

$$
\zeta_{m}(\rho, \beta)=-\beta\left(\zeta_{N} \rho+\ldots\right), \quad \zeta_{N}=\langle p|\bar{u} u-\bar{d} d| p\rangle .
$$

The dots in the rhs of Eqs. (15D) and (16) denote the terms, which are nonlinear in $\rho$. In the gas approximation such terms should be omitted. The $\mathrm{SU}(2)$ invariance of vacuum was assumed in Eq. (16). The expectation value $\kappa_{N}$ is related to the $\pi N$ sigma term $\sigma_{\pi N}$, i.e., [11]

$$
\kappa_{N}=\frac{2 \sigma_{\pi N}}{m_{u}+m_{d}}
$$

with $m_{u, d}$ standing for the current masses of the light quarks, while the value of $\sigma_{\pi N}$ can be extracted from experimental data on low energy $\pi N$ scattering [12, 13]. However, the value $\zeta_{N}$ should be calculated under certain model assumptions on the quark structure of the nucleon. These were the condensates of dimension $d=3$.

Turning to the condensates of dimension $d=4$, we find for the gluon condensate

$$
g_{m}(\rho)=\left\langle M\left|\frac{\alpha_{s}}{\pi} G^{2}(0)\right| M\right\rangle=g_{0}+g(\rho),
$$

$g_{0}=g_{m}(0)$ is the vacuum value,

$$
g(\rho)=g_{N} \rho+\ldots
$$

with the nucleon expectation value

$$
g_{N}=\left\langle N\left|\frac{\alpha_{s}}{\pi} G^{2}\right| N\right\rangle \approx-\frac{8}{9} m,
$$


obtained in [14] in a model-independent way.

Also, the nonlocal condensate $\left\langle N\left|\bar{q}(0) \gamma_{\mu} q(x)\right| N\right\rangle$ provides the contributions of $d=4$ and those of the higher dimension. The term of the dimension $d=4$ is

$$
\left\langle N\left|\bar{q}^{i}(0) \gamma_{\mu} D_{\nu} q^{i}(0)\right| N\right\rangle=\left(g_{\mu \nu}-\frac{4 p_{\mu} p_{\nu}}{m^{2}}\right) m x_{2}
$$

with $x_{2}$ standing for the second moment of the nucleon structure function [9].

The condensates of dimensions $d=3,4$ determine the leading OPE terms of the order $q^{2} \ln q^{2}$ and $\ln q^{2}$ of the lhs of Eq. (17). The next to leading terms of the order $1 / q^{2}$ are determined by the expectation values of the four-quark operators. The importance of these contributions was analyzed in [15]. In the gas approximation they can be presented in terms of the nucleon expectation values. The latter can be obtained in framework of certain models.

We shall analyze the sum rules in the gas approximation. It is a reasonable starting point, since the nonlinear contributions to the most important scalar condensate $\kappa(\rho)$ are relatively small at the densities of the order of the phenomenological saturation density $\rho_{0}=0.17 \mathrm{fm}^{-3}$ of the symmetric matter [10]. The QCD sum rules approach, applied to the description of the nucleon self-energies in the symmetric matter [16] provided the results which are consistent with those, obtained by the methods of nuclear physics.

In the case of symmetric matter $(\beta=0)$, the leading OPE terms $(d=3,4)$ can be either calculated or expressed in terms of the observables. In the asymmetric case we need a model for the calculation of the expectation value $\zeta_{N}$, defined by Eq. (16). In most of the quark models the nucleon is treated as a system of the valence quarks and the isospin symmetric sea of quark-antiquark pairs. Under this assumption the condensate $\zeta_{N}$ is determined by the valence quarks. In the models with the nonrelativistic valence quarks $\zeta_{N}=1$. In more realistic relativistic models $\zeta_{N}<1$ due to the relativistic reduction.

The calculations of the four-quark condensates require model assumptions on the structure of the nucleon. The complete set of the four-quark condensates was obtained in [17. by using the perturbative chiral quark model (PCQM). The chiral quark model, originally suggested in [18], was developed further in [19]-[21]. In the PCQM the nucleon is treated as a system of relativistic valence quarks moving in an effective static field. The valence quarks are supplemented by a perturbative cloud of pseudoscalar mesons, in agreement with the requirements of the chiral symmetry. This model reflects the main points of the nowadays view on the structure of nucleon. The latter is treated as the system of the valence quark and the sea-quarks, contained in pions. The chiral properties are respected. In [17] the SU(2) version of PCQM, which includes only the pions, have been used. Thus, in the calculations, which include the terms of the order $1 / q^{2}$ of OPE obtained in framework of PCQM we must use the PCQM value $\zeta_{N}=0.54[19]$.

In the rhs of the sum rules we describe the nucleon by the relativistic in-medium propagator 22

$$
G_{N}^{-1}=q_{\mu} \gamma^{\mu}-m-\Sigma
$$


with the total self-energy

$$
\Sigma=q_{\mu} \gamma^{\mu} \Sigma_{q}+p_{\mu} \gamma^{\mu} \frac{\Sigma_{p}}{m}+\Sigma_{s}
$$

We shall use the QCD sum rules for the calculation of the nucleon characteristics

$$
\Sigma_{v}=\frac{\Sigma_{p}}{1-\Sigma_{q}}, \quad m^{*}=\frac{m+\Sigma_{s}}{1-\Sigma_{q}}, \quad \Sigma_{s}^{*}=m^{*}-m,
$$

identified with the vector self-energy, Dirac effective mass and the effective scalar self-energy - see, e.g., 22. Two other parameters, to be determined in the approach are the in-medium shifts of the effective values of the nucleon residue $\delta \lambda_{m}^{2}$ and of the continuum threshold $\delta W_{m}^{2}$. We present also the result for the single-particle potential energies

$$
U=\Sigma_{s}^{*}+\Sigma_{v} .
$$

We trace the dependence of these characteristics on the total density $\rho$ and on the asymmetry parameter $\beta$ - Eqs. (11) and (2).

Our approach includes only the strong interactions between the nucleons. The electromagnetic and weak interactions are neglected.

In the asymmetric matter the characteristics obtain different values for the proton and neutron. Considering the asymmetry parameter in the interval $-1 \leq \beta \leq 1$, one can see that the values of any parameter $P_{N}$ for the proton $(p)$ and neutron $(n)$ are connected as

$$
P_{n}(\beta)=P_{p}(-\beta) .
$$

We shall present all the values for the proton, using Eq. (25) to obtain those for the neutron.

We carry out the calculations in the gas approximation, including only terms linear in $\rho$ in the lhs of the sum rules. Thus we can neglect the Fermi motion of the nucleons of the matter, which manifest themselves in higher order of density, and put

$$
s=4 m^{2}
$$

in Eq. (8) . Having in mind future extensions of the approach, we shall keep the dependence on $s$, using Eq. (26) for the specific computations.

In the simplest Hartree approximation (without multiparticle forces) $\Sigma_{p}$ is linear in density. If one neglects the Fermi motion of the nucleon, as we did, the same is true for the scalar self-energy $\Sigma_{s}$. Thus, the parameters $\Sigma_{v}$ and $m^{*}$ (23) exhibit nonlinear behavior due to nonzero value of $\Sigma_{q}\left(\Sigma_{q}=0\right.$ in the mean field approximation). In similar way, the characteristics $\Sigma_{v}$ and $m^{*}$ are not linear in $\rho$ and $\beta$ in our approach due to the relativity large lhs of the sum rules for the structure $\Pi_{m}^{q}$. This reflects distribution of the baryon charge between the pole and continuum. Another reason of the nonlinear effects (although, numerically less important) is the nonlinear structure of the sum rules equations.

The in-medium nucleon QCD sum rules present nucleon interactions with matter in terms of the exchange by uncorrelated quark-antiquark pairs. The latter exchanges correspond to 
the meson exchanges with the same quantum numbers. Thus the contributions of the lowest order OPE terms can be viewed to the exchange by the isoscalar and isovector vector and scalar mesons, with the point-like vertices of the interactions, having the standard Lorentz structure. Inclusion of the nonlocal effects in the quark condensates corresponds to inclusion of nonlocal effects in the vertices of the interactions between the nucleons of the matter and the mesons. The four-quark condensates, in which two of the quark operators are averaged over the vacuum state correspond to the anomalous Lorentz structures of the vertices of the nucleon-meson interactions. In terms of hadronic degrees of freedom the four-quarks condensates, in which all the quark operators act inside the nucleons correspond to the exchanges by the strongly correlated four-quark systems. Such exchanges can be viewed, e.g., as those by two mesons, interacting with the nucleon at the same point. This is illustrated by Figs. 1 and 2 .

We compare our results for the nucleon self-energies and potential energies with those, obtained by the traditional methods of nuclear physics. We find a promising consistency of the results.

We recall in brief the main points of QCD sum rules approach in vacuum and in nuclear matter in Sec. II. We present the general equations in Sec. III, providing the solutions in Sec. IV. We compare the results to those, obtained by the methods of nuclear physics in Sec. V. We summarize in Sec. VI.

\section{GENERAL EQUATIONS}

\section{A. Sum rules in vacuum}

To make the paper self-consistent, we recall the main points of the QCD sum approach in vacuum [1, 2]. The function $\Pi_{0}\left(q^{2}\right)$ (often referred to as "polarization operator") is presented as

$$
\Pi_{0}(q)=i \int d^{4} x e^{i(q x)}\langle 0|T j(x) \bar{j}(0)| 0\rangle
$$

with $j$ being the three-quark local operator (often referred to as "current") with the proton quantum numbers. The usual choice is [2]

$$
j(x)=\varepsilon^{a b c}\left[u^{a T}(x) C \gamma_{\mu} u^{b}(x)\right] \gamma_{5} \gamma_{\mu} d^{c}(x),
$$

where $T$ denotes a transpose and $C$ is the charge conjugation matrix. The upper indices denote the colors.

The lhs of Eq. (4) is approximated by several lowest terms of OPE, i.e., $\Pi_{0}^{j}\left(q^{2}\right) \approx$

$\Pi_{0}^{j, O P E}\left(q^{2}\right)$. The empirical data are used for the spectral function $\operatorname{Im} \Pi_{0}\left(q^{2}\right)$ on the rhs of Eq. (44). Namely, it is known, that the lowest laying state is the bound state of three quarks, which manifests itself as a pole in the (unknown) point $k^{2}=m^{2}$. Since the next singularity is the branching point $k^{2}=W_{p h}^{2}=\left(m+m_{\pi}\right)^{2}$, one can present

$$
\operatorname{Im} \Pi_{0}^{j}\left(k^{2}\right)=\lambda_{N}^{2} a_{j} \delta\left(k^{2}-m^{2}\right)+f^{j}\left(k^{2}\right) \theta\left(k^{2}-W_{p h}^{2}\right)
$$


with $\lambda_{N}^{2}$ being the residue at the nucleon pole. Here $j=q, I$ correspond to the two Lorentz structures of Eq. (3), $a_{q}=1, a_{I}=m$. Thus, Eq. (4) takes the form

$$
\Pi_{0}^{j, O P E}\left(q^{2}\right)=\frac{\lambda_{N}^{2} a_{j}}{m^{2}-q^{2}}+\frac{1}{\pi} \int_{W_{p h}^{2}}^{\infty} \frac{f^{j}\left(k^{2}\right)}{k^{2}-q^{2}} d k^{2} .
$$

Of course, the detailed structure of the spectral density $f^{j}\left(k^{2}\right)$ cannot be resolved in such approach. The further approximations are based on the asymptotic behavior

$$
f^{j}\left(k^{2}\right)=\frac{1}{2 i} \Delta \Pi_{0}^{j, O P E}\left(k^{2}\right)
$$

at $k^{2} \gg\left|q^{2}\right|$ with $\Delta$ denoting the discontinuity. The discontinuity is caused by the logarithmic contributions of the perturbative OPE terms. The usual anzats consists in extrapolation of Eq. (31) to all the values of $k^{2}$, replacing also the physical threshold $W_{p h}^{2}$ by the unknown effective threshold $W_{0}^{2}$, i.e.,

$$
\frac{1}{\pi} \int_{W_{p h}^{2}}^{\infty} \frac{f^{j}\left(k^{2}\right)}{k^{2}-q^{2}} d k^{2}=\frac{1}{2 \pi i} \int_{W_{0}^{2}}^{\infty} \frac{\Delta \Pi_{0}^{j, O P E}\left(k^{2}\right)}{k^{2}-q^{2}} d k^{2} .
$$

Thus Eq. (4) takes the form

$$
\Pi_{0}^{j, O P E}\left(q^{2}\right)=\frac{\lambda_{N}^{2} a_{j}}{m^{2}-q^{2}}+\frac{1}{2 \pi i} \int_{W_{0}^{2}}^{\infty} \frac{\Delta \Pi_{0}^{j, O P E}\left(k^{2}\right)}{k^{2}-q^{2}} d k^{2}
$$

The lhs of Eq. (33) contains QCD condensates. The rhs of Eq. (33) contains three unknown parameters: $m, \lambda_{N}^{2}$ and $W_{0}^{2}$. The OPE becomes increasingly true while the value $\left|q^{2}\right|$ increases. The "pole + continuum" model is more accurate at the smaller values of $\left|q^{2}\right|$. Thus one can expect Eq. (33) to be true in a certain limited interval of the values of $\left|q^{2}\right|$. To improve the overlap of OPE and phenomenological descriptions one usually applies the Borel transform defined as

$$
\begin{aligned}
& B f\left(q^{2}\right)=\lim _{Q^{2}, n \rightarrow \infty} \frac{\left(Q^{2}\right)^{n+1}}{n !}\left(-\frac{d}{d Q^{2}}\right)^{n} f\left(q^{2}\right) \equiv \tilde{f}\left(M^{2}\right), \\
& Q^{2}=-q^{2}, \quad M^{2}=Q^{2} / n
\end{aligned}
$$

with $M$ called the Borel mass. It is important in the applications to the sum rules that Borel transform eliminates the polynomials and emphasizes the contribution of the lowest state in rhs of Eq. (33) due to relation

$$
B \frac{1}{m^{2}-q^{2}}=e^{-m^{2} / M^{2}}
$$


The Borel-transformed form of Eq. (33) reads

$$
\widetilde{\Pi}_{0}^{j, O P E}\left(M^{2}\right)=\lambda_{N}^{2} e^{-m^{2} / M^{2}} a_{j}+\frac{1}{2 \pi i} \int_{W_{0}^{2}}^{\infty} d k^{2} e^{-k^{2} / M^{2}} \Delta \Pi_{0}^{j, O P E}\left(k^{2}\right)
$$

and is known as QCD sum rules. Actually, there are two sum rules for the structures $\Pi_{0}^{q}$ and $\Pi_{0}^{I}$ of the function $\Pi_{0}(q)$ defined by Eq. (3).

It appeared to be more convenient to work with Eq. (36) multiplied by the numerical factor $32 \pi^{4}$. The two sum rules for the nucleon in vacuum can be presented in the form [2]

$$
\begin{aligned}
L_{0}^{q}\left(M^{2}, W_{0}^{2}\right) & =\Lambda_{0}\left(M^{2}\right), \\
L_{0}^{I}\left(M^{2}, W_{0}^{2}\right) & =m \Lambda_{0}\left(M^{2}\right)
\end{aligned}
$$

with

$$
\Lambda_{0}\left(M^{2}\right)=\lambda_{0}^{2} e^{-m^{2} / M^{2}}
$$

Here $\lambda_{0}^{2}=32 \pi^{4} \lambda_{N}^{2}$,

$$
\begin{aligned}
& L_{0}^{q}\left(M^{2}, W_{0}^{2}\right)=32 \pi^{4}\left(\widetilde{\Pi}_{0}^{q, O P E}\left(M^{2}\right)-\frac{1}{2 \pi i} \int_{W_{0}^{2}}^{\infty} d k^{2} e^{-k^{2} / M^{2}} \Delta \Pi_{0}^{q, O P E}\left(k^{2}\right)\right), \\
& L_{0}^{I}\left(M^{2}, W_{0}^{2}\right)=32 \pi^{4}\left(\widetilde{\Pi}_{0}^{I, O P E}\left(M^{2}\right)-\frac{1}{2 \pi i} \int_{W_{0}^{2}}^{\infty} d k^{2} e^{-k^{2} / M^{2}} \Delta \Pi_{0}^{I, O P E}\left(k^{2}\right)\right) .
\end{aligned}
$$

The lhs of Eqs. (37) and (38) 2, 3] have been obtained with the account of the condensates of dimension $d=8$, i.e., with the account of the terms of the order $1 / q^{4}$ in OPE of the functions $\Pi_{0}^{j, O P E}$

$$
\begin{aligned}
& L_{0}^{q}\left(M^{2}, W_{0}^{2}\right)=\frac{M^{6} E_{2}}{L^{4 / 9}}+\frac{b E_{0} M^{2}}{4 L^{4 / 9}}+\frac{4}{3} a^{2} L^{4 / 9}-\frac{1}{3} \frac{\mu_{0}^{2}}{M^{2}} a^{2}, \\
& L_{0}^{I}\left(M^{2}, W_{0}^{2}\right)=2 a M^{4} E_{1}-\frac{a b}{12}+\frac{272}{81} \frac{1}{M^{2}} \frac{\alpha_{s}}{\pi} a^{3}
\end{aligned}
$$

with the traditional notations $a=-(2 \pi)^{2}\langle 0|\bar{q} q| 0\rangle=-2 \pi^{2} \kappa_{0}$ (we assumed the isotopic invariance of vacuum $\langle 0|\bar{u} u| 0\rangle=\langle 0|\bar{d} d| 0\rangle=\langle 0|\bar{q} q| 0\rangle), b=(2 \pi)^{2} g_{0}, \mu_{0}^{2}=0.8 \mathrm{GeV}^{2}$. Here $E_{i}$ are the functions of the ratio $W_{0}^{2} / M^{2}: E_{i}=E_{i}\left(W_{0}^{2} / M^{2}\right)$. They are determined by the formulas

$$
E_{0}(x)=1-e^{-x}, \quad E_{1}(x)=1-(1+x) e^{-x}, \quad E_{2}(x)=1-\left(1+x+\frac{x^{2}}{2}\right) e^{-x} .
$$

The factor

$$
L\left(M^{2}\right)=\frac{\ln M^{2} / \Lambda^{2}}{\ln \nu^{2} / \Lambda^{2}}
$$


accounts for the anomalous dimension, i.e., the most important corrections of the order $\alpha_{s}$ enhanced by the "large logarithms". In Eq. (44) $\Lambda=\Lambda_{Q C D}=0.15 \mathrm{GeV}$, while $\nu=0.5 \mathrm{GeV}$ is the normalization point of the characteristic involved. Note that the two last terms on the rhs of Eq. (41) originate from the four-quark condensates $\left\langle 0\left|\bar{u} \Gamma^{X} u \bar{u} \Gamma^{X} u\right| 0\right\rangle$ and can be expressed through the single term $(\langle 0|\bar{q} q| 0\rangle)^{2}$ only in framework of factorization hypothesis [1]- 3]. Also, the last term on the rhs of Eq. (42) is the six-quark condensate, evaluated in the same approximation.

The matching of the lhs and rhs of Eqs. (37) and (38) have been achieved [2, 3] in the domain

$$
0.8 \mathrm{GeV}^{2}<M^{2}<1.4 \mathrm{GeV}^{2}
$$

providing the values of the vacuum parameters

$$
\lambda_{0}^{2}=1.9 \mathrm{GeV}^{6}, \quad W_{0}^{2}=2.2 \mathrm{GeV}^{2}
$$

if $m=0.94 \mathrm{GeV}$.

\section{B. Sum rules in nuclear matter}

The OPE terms of the polarization operator in nuclear matter

$$
\Pi_{m}(q)=i \int d^{4} x e^{i(q x)}\langle M|T j(x) \bar{j}(0)| M\rangle
$$

contains the in-medium values of QCD condensates. Some of these condensates vanish in vacuum, obtaining non-zero values only in medium. The other ones just change their values compared to the vacuum ones.

The spectrum of the function $\Pi_{m}(q)$ is much more complicated, than that of the vacuum function $\Pi_{0}\left(q^{2}\right)$. However, [7]-[10], the spectrum of the function $\Pi_{m}\left(q^{2}, s\right)$ at fixed value of $s$ can be described by the "pole + continuum" model at least until we include the terms of the order $\rho^{2}$ in the OPE of $\Pi_{m}\left(q^{2}, s\right)$.

Using Eqs. (21) - (23), we present the nucleon propagator as

$$
G_{N}=Z_{N} \cdot \frac{q_{\mu} \gamma^{\mu}-p_{\mu} \gamma^{\mu}\left(\Sigma_{v} / m\right)+m^{*}}{q^{2}-m_{m}^{2}}
$$

with $\Sigma_{v}$ and $m^{*}$ defined by Eq. (23), while the new position of the nucleon pole is

$$
m_{m}^{2}=\frac{\left(s-m^{2}\right) \Sigma_{v} / m-\Sigma_{v}^{2}+m^{* 2}}{1+\Sigma_{v} / m},
$$

and

$$
Z_{N}=\frac{1}{\left(1-\Sigma_{q}\right)\left(1+\Sigma_{v} / m\right)}
$$


Thus, we shall present the dispersion relations for the functions $\Pi_{m}^{j}\left(q^{2}, s\right)(j=q, p, I)$ in the form

$$
\Pi_{m}^{j, O P E}\left(q^{2}, s\right)=\frac{Z_{N} \lambda_{m}^{2} b_{j}}{m_{m}^{2}-q^{2}}+\frac{1}{2 \pi i} \int_{W_{m}^{2}}^{\infty} \frac{\Delta_{k^{2}} \Pi^{j, O P E}\left(k^{2}, s\right)}{k^{2}-q^{2}}
$$

with $b_{q}=1, b_{p}=-\Sigma_{v}, b_{I}=m^{*}$. The Borel-transformed sum rules take the form

$$
\begin{aligned}
& L_{m}^{q}\left(M^{2}, W_{m}^{2}\right)=\Lambda_{m}\left(M^{2}\right), \\
& L_{m}^{p}\left(M^{2}, W_{m}^{2}\right)=-\Sigma_{v} \Lambda_{m}\left(M^{2}\right), \\
& L_{m}^{I}\left(M^{2}, W_{m}^{2}\right)=m^{*} \Lambda_{m}\left(M^{2}\right)
\end{aligned}
$$

with

$$
\Lambda_{m}\left(M^{2}\right)=\lambda_{m}^{* 2} e^{-m_{m}^{2} / M^{2}}
$$

Here

$$
\lambda_{m}^{* 2}=\lambda_{m}^{2} \cdot Z_{N}
$$

is the effective value of the nucleon residue in nuclear matter.

Actually, we shall solve the sum rules equations, subtracting the vacuum effects

$$
\begin{aligned}
& L^{q}\left(M^{2}, W_{m}^{2}, W_{0}^{2}\right)=\Lambda_{m}\left(M^{2}\right)-\Lambda_{0}\left(M^{2}\right) \\
& L^{p}\left(M^{2}, W_{m}^{2}\right)=-\Sigma_{v} \Lambda_{m}\left(M^{2}\right) \\
& L^{s}\left(M^{2}, W_{m}^{2}, W_{0}^{2}\right)=m^{*} \Lambda_{m}\left(M^{2}\right)-m \Lambda_{0}\left(M^{2}\right)
\end{aligned}
$$

with $L^{j}=L_{m}^{j}-L_{0}^{j}$. The functions $\Lambda_{0}, L_{0}^{q}$ and $L_{0}^{I}$ are defined by Eqs. (39), (41) and (42), while $L_{0}^{p}=0$.

\section{CONTRIBUTIONS TO THE LEFT-HAND SIDE OF THE SUM RULES}

We shall use the current, presented by Eq. (28) for the calculation of the function $\Pi_{m}(q)$, defined by Eq. (47). The possibility of using it in isospin asymmetric system requires additional argumentation, which is given in Appendix A.

We shall include the terms of the order $q^{2} \ln q^{2}, \ln q^{2}$ and $1 / q^{2}$ in the lhs of the sum rules (51). This corresponds to the terms of the order $M^{4}, M^{2}$ and $\mathbf{1}$ in the lhs of the Borel transformed equations (57)-(59). We shall include subsequently the contributions of three types. The terms $\ell_{m}^{j}\left(M^{2}\right)$ stand for the lowest order local condensates. They contribute to $q^{2} \ln q^{2}$ and $\ln q^{2}$ terms of Eq. (51). These contributions are similar to simple exchanges by isovector vector and scalar mesons between the nucleon on the rhs of Eq. (151) and the nucleons of the matter, Figs. 1(a),2(a). The terms $u_{m}^{j}\left(M^{2}\right)$ are caused by the nonlocalities of the vector condensate, Fig. 1(d), contributing to the terms of the order $\ln q^{2}$ and $1 / q^{2}$. They correspond to the account of the form factors in the vertices between the isovector mesons couple to the nucleons, Fig. 2(d). Finally, $\omega^{j}\left(M^{2}\right)$ describes the contributions of the four-quark condensates, Figs. 1(b),1(c), being of the order $1 / q^{2}$. They correspond to 
the two-meson exchanges (or to exchanges by four-quark mesons, if there are any) and to somewhat more complicated structure of the meson-nucleon vertices, Fig. 2(c). Thus we present the lhs of Eqs. (152)-(54) as

$$
L_{m}^{j}=\ell_{m}^{j}+u_{m}^{j}+w_{m}^{j}
$$

and the lhs of Eqs. (57)-(59)

$$
L^{j}=\ell^{j}+u^{j}+w^{j}
$$

with $\ell^{j}=\ell_{m}^{j}-\ell_{0}^{j}, u^{j}=u_{m}^{j}, \omega^{j}=\omega_{m}^{j}-\omega_{0}^{j}$, while $\ell_{0}^{j}$ and $\omega_{0}^{j}$ are the corresponding contributions in the vacuum case.

The calculation of the function $\Pi_{m}\left(q^{2}, s\right)$ defined by Eq. (47) is based on the presentation of the single-quark propagator in medium

$$
\left\langle M\left|T q_{\alpha}^{i}(x) \bar{q}_{\beta}^{i}(0)\right| M\right\rangle=G_{\alpha \beta}(x)-\frac{1}{4}\left\langle M\left|\bar{q}^{i}(0) \gamma_{\mu} q^{i}(x)\right| M\right\rangle \gamma_{\alpha \beta}^{\mu}-\frac{1}{4}\left\langle M\left|\bar{q}^{i}(0) q^{i}(x)\right| M\right\rangle \delta_{\alpha \beta}
$$

with $G(x)=\left(i x_{\mu} \gamma^{\mu}\right) /\left(2 \pi^{2} x^{4}\right)$ being the free propagator of the quark in the chiral limit. In the OPE calculations we neglect the values of the current masses of the light quarks since they are small in our scale. Recall that $i$ denotes the light quark flavor. In the lowest orders of OPE two of the quarks are described by the free propagators and only one of the quarks is presented by the second or the third term of the rhs of Eq. (62). Inclusion of the higher order OPE terms will require the two-quark in-medium propagators.

At $x=0$ the matrix elements in the second and third terms on the rhs are just the vector and scalar condensates defined by Eqs. (9) and (15). The contribution of the bilocal configurations can be expressed in terms of the higher moments and twists of the nucleon structure functions [9].

The bilocal operators on the rhs of Eq. (62) are not gauge invariant. The gauge invariant expression, achieved by substitution [23]

$$
q^{i}(x)=q^{i}(0)+x_{\alpha} D_{\alpha} q^{i}(0)+\frac{1}{2} x_{\alpha} x_{\beta} D_{\alpha} D_{\beta} q^{i}(0)+\cdots
$$

with $D_{\alpha}$ standing for the covariant derivatives, provides the infinite set of the local condensates. The expectation values in Eq. (62) depend on the variables $(p x)$ and $x^{2}$. In the gas approximation we need only the nucleon matrix elements. For the vector structure the general form is

$$
\theta_{\mu}^{i}(x)=\left\langle M\left|\bar{q}^{i}(0) \gamma_{\mu} q^{i}(x)\right| M\right\rangle=\frac{p_{\mu}}{m} \Phi_{a}^{i}\left((p x), x^{2}\right)+i x_{\mu} m \Phi_{b}^{i}\left((p x), x^{2}\right)
$$

with $q^{i}(x)$ defined by Eq. (63).

The contribution of the vector structure (64) to the polarization operator $\Pi_{m}(q)$ can be presented as

$$
\Pi_{m}^{V}(q)=\frac{4 i}{\pi^{4}} \int \frac{d^{4} x}{x^{8}}\left(x^{2} \hat{\theta}^{u}+\left(x, \theta^{u}+\theta^{d}\right) \hat{x}\right) e^{i(q x)}
$$


Here we denoted

$$
\hat{a}=a_{\mu} \gamma^{\mu}
$$

for any 4 -vector $a_{\mu}$. For the scalar structure

$$
\Pi_{m}^{I}(q)=-\frac{2 i}{\pi^{4}} \int \frac{d^{4} x}{x^{6}}\langle M|\bar{d}(0) d(x)| M\rangle e^{i(q x)} .
$$

\section{A. Local condensates of the lowest dimensions}

To include the local vector and scalar quark condensates of the lowest dimensions, we must put $x=0$ in Eq. (64) and in the matrix element $\langle M|\bar{d}(0) d(x)| M\rangle$ in Eq. (67). Thus

$$
\Phi_{a}^{i}(0,0)=\left\langle n\left|\bar{q}^{i} \gamma_{0} q^{i}\right| n\right\rangle \rho_{n}+\left\langle p\left|\bar{q}^{i} \gamma_{0} q^{i}\right| p\right\rangle \rho_{p},
$$

while

$$
\langle M|\bar{d}(0) d(0)| M\rangle=\langle 0|\bar{d} d| 0\rangle+\rho_{p}\langle p|\bar{d} d| p\rangle+\rho_{u}\langle n|\bar{d} d| n\rangle .
$$

We must add also the contribution of the gluon condensate. Technically it can be obtained by replacing the vacuum gluon condensate by it's in-medium value - Eqs. (18) and (19).

The contributions of these local condensates can be expressed as

$$
\begin{aligned}
\ell^{q} & =f_{v}^{q}\left(M^{2}, W_{m}^{2}\right) v^{q}(\rho)+f_{g}^{q}\left(M^{2}, W_{m}^{2}\right) g(\rho), \\
\ell^{p} & =f_{v}^{p}\left(M^{2}, W_{m}^{2}\right) v^{p}(\rho, \beta), \\
\ell^{I} & =f_{\kappa}^{I}\left(M^{2}, W_{m}^{2}\right) t^{I}(\rho, \beta),
\end{aligned}
$$

with the dependence on $\rho$ and $\beta$ being contained in the factors

$$
v^{q}(\rho)=3 \rho, \quad v^{p}(\rho, \beta)=3 \rho\left(1-\frac{\beta}{4}\right), \quad t^{I}(\rho, \beta)=\rho\left(\kappa_{N}+\zeta_{N} \beta\right),
$$

and the function $g(\rho)$, given by Eqs. (18) and (19). The other functions are [16]

$$
\begin{aligned}
f_{v}^{q}\left(M^{2}, W_{m}^{2}\right) & =-\frac{8 \pi^{2}}{3} \frac{\left(s-m^{2}\right) M^{2} E_{0 m}-M^{4} E_{1 m}}{m L^{4 / 9}}, \\
f_{g}^{q}\left(M^{2}, W_{m}^{2}\right) & =\frac{\pi^{2} M^{2} E_{0 m}}{L^{4 / 9}}, \\
f_{v}^{p}\left(M^{2}, W_{m}^{2}\right) & =-\frac{8 \pi^{2}}{3} \frac{4 M^{4} E_{1 m}}{L^{4 / 9}} \\
f_{\kappa}^{I}\left(M^{2}, W_{m}^{2}\right) & =-4 \pi^{2} M^{4} E_{1 m} .
\end{aligned}
$$

The notation $E_{k m}(k=0,1)$, Eq. (43), means that the functions depend on the ratio $W_{m}^{2} / M^{2}$. 
Now we must find the $\beta$-dependence of the nucleon self-energies and also of the parameters $W_{m}^{2}$ and $\lambda_{m}^{* 2}$. Note that there is a simple solution of Eqs. (52) and (54)

$$
\begin{aligned}
& \Sigma_{v}(\rho, \beta)=\Sigma_{v}(\rho, 0)\left(1-\frac{\beta}{4}\right) \\
& m^{*}\left(\rho, \beta ; \kappa_{N}, \zeta_{N}\right)=m^{*}\left(\rho, 0 ; \kappa_{N}+\beta \zeta_{N}, 0\right) \\
& W_{m}^{2}(\rho, \beta)=W_{m}^{2}(\rho, 0)
\end{aligned}
$$

which is true with the same accuracy as the solutions for the symmetric matter $(\beta=0)[16$. Indeed, assuming that $W_{m}^{2}$ does not change with $\beta$ (73) , we find that the function $\Lambda_{m}\left(M^{2}\right)$ in the lhs of Eq. (52) should not depend on $\beta^{1}$. This leads to Eqs. (74) and (75).

\section{B. Inclusion of the nonlocal condensates}

Now we present the functions in the rhs of Eq. (64) as

$$
\Phi_{a(b)}^{i}=\rho_{n} \phi_{n, a(b)}^{i}+\rho_{p} \phi_{p, a(b)}^{i} .
$$

Due to $\mathrm{SU}(2)$ invariance they can be presented in terms of the proton functions $\phi_{a(b)}^{i}=\phi_{p, a(b)}^{i}$

$$
\Phi_{a(b)}^{i}=\rho_{n} \phi_{a(b)}^{i^{\prime}}+\rho_{p} \phi_{a(b)}^{i}
$$

with $i=u, d, i^{\prime} \neq i$.

Expansion in powers of $x^{2}$ corresponds to the expansion of the function $\Pi_{m}(q)$ in powers of $q^{2}$. To obtain the terms of the order $q^{-2}$ it is sufficient to include two lowest terms of the expansions in powers of $x^{2}$. One can present [9, 24]

$$
\phi_{a(b)}^{i}\left((p x), x^{2}\right)=\int_{0}^{1} d \alpha e^{-i \alpha(p x)} f_{a(b)}^{i}\left(\alpha, x^{2}\right)
$$

with

$$
f_{a(b)}^{i}\left(\alpha, x^{2}\right)=\eta_{a(b)}^{i}(\alpha)+\frac{1}{8} x^{2} m^{2} \xi_{a(b)}^{i}(\alpha)
$$

Here $\eta_{a}^{i}(\alpha)=f_{a}^{i}(\alpha, 0)$ is the contribution of the quarks with the flavor $i$ to the asymptotic of the nucleon structure function $\eta(\alpha)=\eta_{a}^{u}(\alpha)+\eta_{a}^{d}(\alpha)$, normalized by the condition

$$
\int_{0}^{1} d \alpha \eta(\alpha)=3
$$

with the rhs presenting just the number of the valence quarks in the nucleon. Thus, expansion of the function

$$
\varphi_{a}^{i}(p x)=\phi_{a}^{i}((p x), 0)
$$

\footnotetext{
${ }^{1}$ The numerical solution, which will be considered in Sec. IV provides indeed $W_{m}^{2}(\rho, \beta) \approx W_{m}^{2}(\rho, 0)$ with the accuracy of $10 \%$.
} 
in powers of $(p x)$ is expressed through the moments of the distributions $\eta_{a}^{i}(\alpha)$. The moments are well known - at least, those, which are numerically important. Also, the first moments

$$
\xi_{a}^{i}=\int_{0}^{1} \xi_{a}^{i}(\alpha) d \alpha
$$

of the distribution $\xi_{a}^{i}(\alpha)$ were calculated in [25] by the QCD sum rules method:

$$
\xi^{u}=-0.24, \quad \xi^{d}=-0.09, \quad \xi=\xi^{u}+\xi^{d}=-0.33 .
$$

The moments of the function $\eta_{b}^{i}(\alpha)$ can be obtained by using the equations of motion $D_{\alpha} \gamma^{\alpha} q^{i}(x)=m_{i} q^{i}(x)$. Thus, in the chiral limit [9]

$$
\begin{aligned}
& \left\langle\varphi_{b}^{i}\right\rangle=\frac{1}{4}\left\langle\varphi_{a}^{i} \alpha\right\rangle, \\
& \left\langle\varphi_{b}^{i} \alpha\right\rangle=\frac{1}{5}\left(\left\langle\varphi_{a}^{i} \alpha^{2}\right\rangle-\frac{1}{4}\left\langle\xi^{i}\right\rangle\right), \\
& \left\langle\xi_{b}^{i}\right\rangle=\frac{1}{6}\left\langle\xi_{a}^{i} \alpha\right\rangle .
\end{aligned}
$$

Here we denoted

$$
\langle f\rangle=\int_{0}^{1} d \alpha f(\alpha)
$$

for any function $f(\alpha)$.

Note that the nonlocality of the scalar condensate, i.e., of the last term on the rhs of Eq. (62) does not manifest itself in the terms up to $1 / q^{2}$. The first derivative in $(p x)$, as well all the derivatives of the odd order vanish in the chiral limit due to QCD equation of motion. The next to leading order of the expansion in powers of $x^{2}$ vanishes due to certain cancellations [8] as well as in the case of vacuum [2] for the particular choice of the operator $j(x)$ presented by Eq. (28). We neglect the terms, containing more complicated gluon condensates, since they are numerically small [26].

Using Eqs. (64) and (77) we obtain for the vector structure (65)

$$
\begin{aligned}
& \Pi_{m}^{V}(q)=\Pi_{m}^{V a}(q)+\Pi_{m}^{V b}(q), \\
& \Pi_{m}^{V a}(q)=\frac{2 i}{\pi^{4} m}\left\{\int \frac{d^{4} x}{x^{8}}\left(\hat{p} x^{2}+2 \hat{x}(p x)\right)\left(\varphi_{a}^{u}+\varphi_{u}^{d}\right) e^{i(q x)} \rho\right. \\
& \left.-\beta \hat{p} \int \frac{d^{4} x}{x^{6}}\left(\varphi_{a}^{u}-\varphi_{a}^{d}\right) e^{i(q x)} \rho\right\}, \\
& \Pi_{m}^{V b}(q)=-\frac{6 m}{\pi^{4}} \int \frac{d^{4} x}{x^{6}} \hat{x}\left(\varphi_{b}^{u}+\varphi_{b}^{d}\right) e^{i(q x)} \rho+\frac{2 \beta}{\pi^{4}} \int \frac{d^{4} x}{x^{6}} \hat{x}\left(\varphi_{b}^{u}-\varphi_{b}^{d}\right) e^{i(q x)} .
\end{aligned}
$$

The further calculations, employing Eqs. (78) and (79) are similar to those, carried out for the case of symmetric matter [16 — see Appendix B. 
Finally, the higher moments and higher twists of the nucleon structure functions provide the contributions $u^{i}$ to the lhs $L_{m}^{i}$ of the sum rules - Eq. (60)

$$
\begin{aligned}
& u^{q}\left(M^{2}\right)=\left(u_{N, 1}^{q}\left(M^{2}\right)+\beta u_{N, 2}^{q}\left(M^{2}\right)\right) \rho, \\
& u_{N, 1}^{q}\left(M^{2}\right)=\frac{8 \pi^{2}}{3 L^{4 / 9} m}\left[-\frac{5}{2} m^{2} M^{2} E_{0 m}\langle\eta \alpha\rangle+\frac{3}{2} m^{2}\left(s-m^{2}\right)\langle\xi\rangle\right], \\
& u_{N, 2}^{q}\left(M^{2}\right)=\frac{8 \pi^{2}}{3 L^{4 / 9} m}\left[\frac{3}{2} m^{2} M^{2} E_{0 m}\left(\left\langle\eta^{u} \alpha\right\rangle-\left\langle\eta^{d} \alpha\right\rangle\right)\right] ; \\
& u^{p}\left(M^{2}\right)=\left(u_{N, 1}^{p}\left(M^{2}\right)+\beta u_{N, 2}^{p}\left(M^{2}\right)\right) \rho, \\
& u_{N, 1}^{p}\left(M^{2}\right)=\frac{8 \pi^{2}}{3 L^{4 / 9}}\left[-5\left(M^{4} E_{1 m}-\left(s-m^{2}\right) M^{2} E_{0 m}\right)\langle\eta \alpha\rangle\right. \\
& \left.-\frac{12}{5} m^{2} M^{2} E_{0 m}\left\langle\eta \alpha^{2}\right\rangle+\frac{18}{5} m^{2} M^{2} E_{0 m}\langle\xi\rangle\right], \\
& u_{N, 2}^{p}\left(M^{2}\right)=\frac{8 \pi^{2}}{3 L^{4 / 9}}\left[3\left(M^{4} E_{1 m}-\left(s-m^{2}\right) M^{2} E_{0 m}\right)\left\langle\left(\eta^{u}-\eta^{d}\right) \alpha\right\rangle\right. \\
& \left.+\frac{9}{5} m^{2} M^{2} E_{0 m}\left\langle\left(\eta^{u}-\eta^{d}\right) \alpha^{2}\right\rangle-\frac{27}{10} m^{2} M^{2} E_{0 m}\left\langle\left(\xi^{u}-\xi^{d}\right)\right\rangle\right] ; \\
& u^{I}\left(M^{2}\right)=0 .
\end{aligned}
$$

Here we denote $L=L\left(M^{2}\right), E_{1 m}=E_{1}\left(W_{m}^{2} / M^{2}\right), E_{0 m}=E_{0}\left(W_{m}^{2} / M^{2}\right)$, Eq. (43), the value of $\xi$ is determined by Eq. (81).

\section{Inclusion of the four-quark condensates}

The exchange by two quark-antiquark pairs between the current (28) and the matter is described in terms of the four-quark expectation values

$$
H_{m}^{X Y}(\rho)=\left\langle M\left|\bar{u} \Gamma^{X} u \bar{u} \Gamma^{Y} u\right| M\right\rangle \text { and } R_{m}^{X Y}(\rho)=\left\langle M\left|\bar{d} \Gamma^{X} d \bar{u} \Gamma^{Y} u\right| M\right\rangle
$$

with $\Gamma^{X, Y}$ being the basic $4 \times 4$ matrices

$$
\Gamma^{I}=I, \quad \Gamma^{P s}=\gamma_{5}, \quad \Gamma^{V}=\gamma_{\mu}, \quad \Gamma^{A}=\gamma_{\mu} \gamma_{5}, \quad \Gamma^{T}=\frac{i}{2}\left(\gamma_{\mu} \gamma_{\nu}-\gamma_{\nu} \gamma_{\mu}\right),
$$

acting on the Lorentz indices of the quark operators. We did not display the color indices in Eq. (88), keeping in mind that the quark operators are color antisymmetric.

In the gas approximation

$$
\begin{aligned}
& H_{m}^{X Y}(\rho, \beta)=H_{m}^{X Y}(0)+\rho_{n} h_{n}^{X Y}+\rho_{p} h_{p}^{X Y} \\
& R_{m}^{X Y}(\rho, \beta)=R_{m}^{X Y}(0)+\rho_{n} r_{n}^{X Y}+\rho_{p} r_{p}^{X Y}
\end{aligned}
$$


The characteristics $h_{N}^{X Y}$ and $r_{N}^{X Y}$ can be presented as

$$
\begin{aligned}
h_{N}^{X Y} & =\frac{5}{6}\left(\left\langle 0\left|\bar{u} \Gamma^{X} u\right| 0\right\rangle\left\langle N\left|\bar{u} \Gamma^{Y} u\right| N\right\rangle+\left\langle 0\left|\bar{u} \Gamma^{Y} u\right| 0\right\rangle\left\langle N\left|\bar{u} \Gamma^{X} u\right| N\right\rangle\right) \\
& +\left\langle N\left|\left(\bar{u} \Gamma^{X} u \cdot \bar{u} \Gamma^{Y} u\right)_{i n t}\right| N\right\rangle, \\
r_{N}^{X Y} & =\frac{2}{3}\left(\left\langle 0\left|\bar{d} \Gamma^{X} d\right| 0\right\rangle\left\langle N\left|\bar{u} \Gamma^{Y} u\right| N\right\rangle+\left\langle 0\left|\bar{u} \Gamma^{Y} u\right| 0\right\rangle\left\langle N\left|\bar{d} \Gamma^{X} d\right| N\right\rangle\right) \\
& +\left\langle N\left|\left(\bar{d} \Gamma^{X} d \cdot \bar{u} \Gamma^{Y} u\right)_{\text {int }}\right| N\right\rangle .
\end{aligned}
$$

The first two ("factorized") terms in the rhs of Eqs. (91) and (92) describe two quark operators, acting on the vacuum state, while the other two operators act inside the nucleon. The last terms describe the "internal" action of all four operators inside the nucleon. This is shown by the lower index "int". The coefficients $5 / 6$ and $2 / 3$ on rhs of Eqs. (91) and (92) present the weights of the color-antisymmetric states [16].

The contribution of the four-quark expectation values to the in-medium change of the polarization operator can be written as

$$
\begin{aligned}
& (\Pi)_{4 q}=\left(\Pi_{m}\right)_{4 q}-\left(\Pi_{0}\right)_{4 q}=\frac{\rho_{n}}{q^{2}}\left(\sum_{X, Y} \mu_{X Y} h_{n}^{X Y}+\sum_{X, Y} \tau_{X Y} r_{n}^{X Y}\right) \\
& +\frac{\rho_{p}}{q^{2}}\left(\sum_{X, Y} \mu_{X Y} h_{p}^{X Y}+\sum_{X, Y} \tau_{X Y} r_{p}^{X Y}\right) .
\end{aligned}
$$

The matrices $\mu_{X Y}$ and $\tau_{X Y}$ can be obtained by using the general expression for the function $\Pi_{m}(q)$ presented in $[26]$

$$
\begin{aligned}
\mu_{X Y} & =\frac{\theta_{Y}}{16} \operatorname{Tr}\left(\gamma_{\alpha} \Gamma^{X} \gamma_{\beta} \Gamma^{Y}\right) \gamma_{5} \gamma^{\alpha} \hat{q} \gamma^{\beta} \gamma_{5}, \\
\tau_{X Y} & =\frac{\theta_{Y}}{4} \operatorname{Tr}\left(\gamma_{\alpha} \hat{q} \gamma_{\beta} \Gamma^{Y}\right) \gamma_{5} \gamma^{\alpha} \Gamma^{X} \gamma^{\beta} \gamma_{5}, \quad \hat{q}=q_{\mu} \gamma^{\mu} .
\end{aligned}
$$

Here $\theta_{Y}=1$ if $\Gamma^{Y}$ has a vector or tensor structure, while $\theta_{Y}=-1$ in the scalar, pseudoscalar and axial cases. The sign is determined by that of the commutator between matrix $\Gamma^{Y}$ and the charge conjugation matrix $C$ - Eq. (16).

The products $\mu_{X Y} h_{i}^{X Y}$ obtain nonzero values if the matrices $\Gamma^{X}$ and $\Gamma^{Y}$ have the same Lorentz structure. In this case all the structures presented by Eq. (89) contribute to $\left(\Pi_{m}\right)_{4 q}$. The products $\tau_{X Y} r^{X Y}$ do not turn to zero only if $\Gamma^{Y}$ has a vector or axial structure. In the latter case $\Gamma^{X}$ should be an axial matrix as well. In the former case $\Gamma^{X}$ can be either Lorentz scalar or Lorentz vector.

We denote $h_{i}^{X X}=h_{i}^{X}, \mu_{X X}=\mu_{X}$ and $r_{i}^{X X}=r_{i}^{X}, \tau_{X X}=\tau_{X}$ for the similar Lorentz structures $X$ and $Y$. The scalar and pseudoscalar expectation values are Lorentz scalars. Thus, their contributions can be expressed through single parameters $a^{S}, a^{P s}, a^{S V}$. The latter is true also for the scalar-vector expectation value $r^{S V}$. We obtain

$$
\mu_{S}=-\frac{\hat{q}}{2}, \quad \mu_{P s}=\frac{\hat{q}}{2}, \quad\left(\tau_{S V}\right)_{\mu}=-2 q_{\mu} .
$$


In the other channels the four-quark condensates have more complicated structure. In the vector and axial channels

$$
\begin{aligned}
& \left(h_{i}^{V(A)}\right)_{\mu \nu}=a_{h, i}^{V(A)} g_{\mu \nu}+b_{h, i}^{V(A)} \frac{p_{\mu} p_{\nu}}{m^{2}} \\
& \left(r_{i}^{V(A)}\right)_{\mu \nu}=a_{r, i}^{V(A)} g_{\mu \nu}+b_{r, i}^{V(A)} \frac{p_{\mu} p_{\nu}}{m^{2}}
\end{aligned}
$$

In the tensor channel

$$
\left(h_{i}^{T}\right)_{\mu \nu, \rho \tau}=a_{h, i}^{T} s_{\mu \nu, \rho \tau}+b_{h, i}^{T} t_{\mu \nu, \rho \tau}
$$

with

$$
\begin{aligned}
s_{\mu \nu, \rho \tau} & =g_{\mu \rho} g_{\nu \tau}-g_{\mu \tau} g_{\nu \rho} \\
t_{\mu \nu, \rho \tau} & =\frac{1}{m^{2}}\left(p_{\mu} p_{\rho} g_{\nu \tau}+p_{\nu} p_{\tau} g_{\mu \rho}-p_{\mu} p_{\tau} g_{\nu \rho}-p_{\nu} p_{\rho} g_{\mu \tau}\right) .
\end{aligned}
$$

Using Eq. (94) we obtain

$$
\begin{aligned}
& \mu_{V} h_{i}^{V}=-a_{h, i}^{V} \hat{q}-b_{h, i}^{V} \frac{\hat{p}(p q)}{m^{2}}, \quad \mu_{A} h_{i}^{A}=a_{h, i}^{A} \hat{q}+b_{h, i}^{A} \frac{\hat{p}(p q)}{m^{2}} \\
& \tau_{V i} r_{i}^{V}=\left(-10 a_{r, i}^{V}-2 b_{r, i}^{V}\right) \hat{q}-2 b_{r, i}^{V} \frac{\hat{p}(p q)}{m^{2}} \\
& \tau_{A i} r_{i}^{A}=\left(-6 a_{r, i}^{A}-2 b_{r, i}^{A}\right) \hat{q}+2 b_{r, i}^{A} \frac{\hat{p}(p q)}{m^{2}} \\
& \mu_{T} h_{i}^{T}=b_{h, i}^{T}\left(-\frac{\hat{q}}{2}+\frac{2 \hat{p}(p q)}{m^{2}}\right) .
\end{aligned}
$$

We can write Eq. (93) in the form

$$
(\Pi)_{4 q}=B^{q} \frac{\hat{q}}{q^{2}}+B^{p} \frac{(p q)}{m^{2}} \frac{\hat{p}}{q^{2}}+B^{I} \frac{m I}{q^{2}}
$$

with each of the coefficients $B^{j}$ being the composition of the contributions of the neutrons and protons of the matter $B_{n}^{j}$ and $B_{p}^{j}$, i.e.,

$$
B^{j}=B_{n}^{j} \rho_{n}+B_{p}^{j} \rho_{p}
$$

Using Eqs. (95) - (97) we obtain

$$
\begin{aligned}
B_{i}^{q} & =\left(-\frac{1}{2} a_{h, i}^{S}+\frac{1}{2} a_{h, i}^{P s}-a_{h, i}^{V}+a_{h, i}^{A}-\frac{1}{2} b_{h, i}^{T}\right)-2\left(5 a_{r, i}^{V}+b_{r, i}^{V}+3 a_{r, i}^{A}+b_{r, i}^{A}\right), \\
B_{i}^{p} & =-b_{h, i}^{V}+b_{h, i}^{A}+2 b_{h, i}^{T}-2\left(b_{r, i}^{V}-b_{r, i}^{A}\right), \\
B_{i}^{I} & \approx-2 a_{i}^{S V} \frac{(p q)}{m^{2}} .
\end{aligned}
$$


Here we denoted $a_{i}^{S}=h_{i}^{S S}, a_{i}^{P s}=h_{i}^{P s P s}$. Recall that the lower index " $i$ " stands for the neutron or the proton. In the last equality we defined $r_{i}^{S V}=a_{i}^{S V} p_{\mu} / m$, neglecting the nonlocal contributions to the scalar-vector condensate. However in the scalar channel such terms provide the contributions of the order $1 / q^{2}$ to the rhs of Eq. (100). They will be included below - Appendix C.

The calculations of the contributions to rhs of Eq. (102) require the model assumption on the structure of the nucleon. The complete set of the four-quark condensate was obtained in [17 by using the perturbative chiral quark model (PCQM). The chiral quark model, originally suggested in [18, was developed further in [19]-21]. In the PCQM the nucleon is treated as a system of relativistic valence quarks moving in an effective static field. The valence quarks are supplemented by a perturbative cloud of the pseudoscalar mesons in agreement with the requirements of the chiral symmetry. In [17] the simplest SU(2) version of PCQM, which includes only the pions, have been used. The parameters which enter Eq. (102) are calculated in this version of PCQM.

There are three types of contributions to the four-quark condensate in the framework of this approach. All four operators can act on the valence quarks. Also, four operators can act on the pion. There is also a possibility that two of the operators act on the valence quarks while the other two act on the pions. Following [17] we speak of the "interference terms" in the latter case.

To obtain the contribution of the pion cloud, we need the expectation values of the four-quark operators in pions. The latter have been deduced in [27] by using the current algebra technique. It was shown in [16], that in the case of the symmetric matter there is a remarkable cancellation of the pion contributions in the function $\Pi_{m}(q)$. Since the pion contents of the asymmetric matter manifests itself in the different intensities of $\pi^{+}$and $\pi^{-}$fields, while the four-quark expectation values in $\pi^{+}$and $\pi^{-}$mesons are the same, the cancellation takes place in this case as well. This cancellation takes place in any model of the nucleon which treats the pion cloud perturbatively. Thus, the contributions of the four-quark condensates come from the terms, determined by the valence quarks only and from the interference terms.

This enables us to present Eq. (91) as

$$
h_{i}^{X}=2 \cdot \frac{5}{6}\left\langle 0\left|\bar{u} \Gamma^{X} u\right| 0\right\rangle\left\langle N_{i}\left|\left(\bar{u} \Gamma^{X} u\right)_{v}\right| N_{i}\right\rangle+\left\langle N_{i}\left|\left(\bar{u} \Gamma^{X} u \bar{u} \Gamma^{X} u\right)_{1}\right| N_{i}\right\rangle .
$$

Here the lower index " $v$ " means that the operators act on the valence quarks only. The lower index "1" corresponds to the sum of the term in which all the four operators act on the valence quarks and the term in which two of the operators act on the valence quarks while the other two act on pions. Of course, the first ("factorized") term in the rhs of Eq. (103) obtains a nonvanishing value only in the scalar case $\Gamma^{X}=I$.

The expectation values of the operators of different flavors, providing nonvanishing contributions to the rhs of Eq. (92) are the scalar-vector condensate

$$
r_{i \mu}^{S V}=2 \cdot \frac{2}{3}\langle 0|\bar{d} d| 0\rangle\left\langle N_{i}\left|\bar{u} \gamma_{\mu} u\right| N_{i}\right\rangle+\left\langle N_{i}\left|\left(\bar{d} d \bar{u} \gamma_{\mu} u\right)_{i n t}\right| N_{i}\right\rangle
$$


and

$$
r_{i \mu \nu}^{X}=\left\langle N_{i}\left|\left(\bar{d} \Gamma_{\mu}^{X} d \bar{u} \Gamma_{\nu}^{X} u\right)_{1}\right| N_{i}\right\rangle
$$

with $X$ standing for vector or axial structures. The meaning of the lower index " 1 " is the same as in Eq. (103).

Note that among the interference terms contributing to the four-quark condensates, there is so-called "vertex interference", in which one of the PCQM vertices of the self-energy of the valence quark is replaced by the four-quark operator. Some of such terms contain the matrix elements $\left\langle 0\left|\bar{u} \gamma_{5} d\right| \pi^{-}\right\rangle$and $\left\langle 0\left|\bar{d} \gamma_{5} u\right| \pi^{+}\right\rangle$, contributing to the expectation values $\left\langle N\left|\bar{u} \gamma_{5} d \bar{d} \gamma_{5} u\right| N\right\rangle$, being connected with the matrix elements $\left\langle N\left|\bar{d} \Gamma^{X} d \bar{u} \Gamma^{X} u\right| N\right\rangle$ of all structures $\Gamma^{X}$ by the Fierz transform. On the other hand, they depend on the values of the quark masses, since $\left\langle 0\left|\bar{u} \gamma_{5} d\right| \pi^{-}\right\rangle=-\frac{i \sqrt{2} F_{\pi} M_{\pi}^{2}}{m_{u}+m_{d}}$ with $M_{\pi}$ and $F_{\pi}$ denoting the mass and the decay constant of pion. In a somewhat straightforward approach one substitutes the current quark masses. Following more sophisticated models of the pions [28] one should substitute the constituent quark masses, thus obtaining the values, which are negligibly small in our scale.

Using the complete set of the nucleon four-quark expectation values [17, we obtain

$$
(\Pi)_{4 q}=\left(A_{4 q}^{q}(\beta) \frac{\hat{q}}{q^{2}}+A_{4 q}^{p}(\beta) \frac{(p q)}{m^{2}} \frac{\hat{p}}{q^{2}}+A_{4 q}^{I}(\beta) m \frac{I}{q^{2}}\right) \frac{a}{(2 \pi)^{2}} \rho
$$

with the coefficients $A_{4 q}^{i}(\beta)$ being determined by Eqs. (103)-(105), while

$$
a=-(2 \pi)^{2}\langle 0|\bar{u} u| 0\rangle
$$

We use the value $\langle 0|\bar{u} u| 0\rangle=(-241 \mathrm{MeV})^{3}$, corresponding to $a=0.55 \mathrm{GeV}^{3}$, employed in 3 . Note that $a$ is just a convenient scale for presentation of the results. It does not reflect the chiral properties of $\Pi_{4 q}$.

The $\hat{q}$ term results mainly as the sum of the expectation value of the product of the four $u$-quark operators, described by the first ("factorized") term on the rhs of Eq. (103), and that of the product of two $u$ and two $d$-quark operators in the vector channel - Eq. (105). The former contributions depend on $\beta$, while the latter do not. Thus, the coefficient $A_{4 q}^{q}$ depends on $\beta$ strongly. The $\hat{p}$ term is determined mostly by the expectation value (105) in the vector channel, with the protons and neutrons providing the equal contributions. This explains the weak dependence of the parameter $A_{4 q}^{p}$ on $\beta$. The coefficient $A_{4 q}^{I}$ is dominated by the first term on the rhs of Eq. (104), providing stronger dependence on $\beta$. The calculations give

$$
A_{4 q}^{q}=-0.11-0.21 \beta, \quad A_{4 q}^{p}=-0.57+0.09 \beta, \quad A_{4 q}^{I}=1.90-0.92 \beta .
$$

In the simplified model of the pion, which does not include the renormalization of the quark masses by the interactions, the value of the coefficient $A_{4 q}^{q}$ is somewhat different

$$
A_{4 q}^{q}=0.25-0.22 \beta
$$

while the values of $A_{4 q}^{p}$ and $A_{4 q}^{I}$ remain unchanged. 
The contributions of the four-quark condensates to the lhs of the Borel transformed sum rules (57)-(59) can be presented in the same way, as for the symmetric case

$$
\begin{aligned}
& \omega^{j}=\omega_{N}^{j} \rho, \quad \omega_{N}^{j}=A_{4 q}^{j}(\beta) f_{4 q}^{j}, \\
& f_{4 q}^{q}=-8 \pi^{2} a, \quad f_{4 q}^{p}=-8 \pi^{2} \frac{s-m^{2}}{2 m} a, \quad f_{4 q}^{I}=-8 \pi^{2} m a .
\end{aligned}
$$

\section{SOLUTIONS OF THE SUM RULES EQUATIONS}

Now we present the solutions of the sum rules equations, focusing on the functions $\Sigma_{v}(\rho, \beta)$ and $m^{*}(\rho, \beta)$. We shall include the terms $\ell^{j}, u^{j}$ and $\omega^{j}$ (Eq. (61) $)$ in the succession in lhs of Eqs. (57)-(59). Two lowest order OPE contributions to the vector structures $\hat{q}$ and $\hat{p}$ are presented in terms of the vector and gluon condensates and of the nucleon structure functions. These characteristics are either calculated in a model-independent way, or determined in the experiments. The lowest order OPE terms in the scalar channel are expressed in terms of isotope-symmetric and isotope-asymmetric scalar condensates $\kappa_{N}=\langle p|\bar{u} u+\bar{d} d| p\rangle$ and $\zeta_{N}=\langle p|\bar{u} u-\bar{d} d| p\rangle-$ Eqs. (15) and (16). Here the situation becomes somewhat more complicated.

The expectation value $\kappa_{N}$ is related to the $\pi N$ sigma-term $\sigma_{\pi N}$ by Eq. (17). The value of $\sigma_{\pi N}$ can be extracted from the data on low-energy $\pi N$ scattering. The procedure consists in subtracting the high-order chirality-violating terms $\sigma^{\prime}$ from the experimental value $\Sigma_{\pi N}$, i.e., $\sigma_{\pi N}=\Sigma_{\pi N}-\sigma^{\prime}$. The value $\sigma^{\prime} \approx 15 \mathrm{MeV}$ was obtained in [29] by dispersion relation technique. However, there are some uncertainties in deducing the value of $\Sigma_{\pi N}$ from the experimental data. The canonical value $\Sigma_{\pi N}=(60 \pm 8) \mathrm{MeV}$ [12] is now challenged by the higher values $77 \pm 6 \mathrm{MeV}$ [13]. Assuming $m_{u}+m_{d}=11 \mathrm{eV}$ [11], we find that $\Sigma_{\pi N}=64 \mathrm{MeV}$ corresponds to $\kappa_{N}=8$. Additional uncertainties emerge, since the true value of the sum $m_{u}+m_{d}$ may be somewhat larger.

There is no experimental data on the expectation value $\zeta_{N}$. If the nucleon is treated as a system of the valence quarks and the isospin symmetric sea of the quark-antiquark pairs, the expectation value $\zeta_{N}$ is determined by the contribution of the valence quarks. Thus, the reasonable values are $\zeta_{N}=1$ for the nonrelativistic models and $\zeta_{N}<1$ in the relativistic case. Until we include only the leading OPE terms $\ell^{j}$, we can solve the sum rules equations for any values of $\kappa_{N}$ and $\zeta_{N}$. However the four-quark condensates are obtained in framework of a specific perturbative chiral quark model (PCQM). Within this model $\sigma_{\pi N}=45 \mathrm{MeV}$ [20], leading to $\kappa_{N}=8$. Thus, to be self-consistent, we must use this value as the basic one in the general equations, which include the contributions $\omega^{j}$. Note also, that the values of $\Sigma_{\pi N}$ extracted from the experimental data are correlated with the assumption on the strange quark content $y_{N}=\frac{2\langle p|\bar{s} s| p\rangle}{\langle p|\bar{u} u+d d| p\rangle}\left[13\right.$. The values $\Sigma_{\pi N} \approx 77 \mathrm{MeV}$ correspond to $y_{N} \approx 0.35$, with a large part of the nucleon mass being due to the strange quarks. The smaller values of $\Sigma_{\pi N}$ require much smaller values of $y_{N}$. In PCQM one finds $y_{N}=0.08$ [20], in agreement with the smaller values of $\Sigma_{\pi N}$. The PCQM value $\zeta_{N}=0.54$ can be obtained by using the results of [19]. 
We shall present most of the numerical results for the values

$$
\kappa_{N}=8, \quad \zeta_{N}=0.54
$$

Anyway, in the linearized version of the sum rules, the nucleon characteristics will be presented as the explicit function of the condensates, e.g., of the parameters $\kappa_{N}$ and $\zeta_{N}$.

\section{A. Solution of the general equations}

Here we present the solutions of the general equations (57)-(59), which are identical to Eqs. (52)-(54). Recall that we approximate the in-medium condensates by the functions, which are linear both in $\rho$ and $\beta$. However the solutions $\Sigma_{v}(\rho, \beta)$ and $m^{*}(\rho, \beta)$ are not linear. One can demonstrate this by presenting Eqs. (53) and (54) as

$$
\Sigma_{v}=-\frac{L_{m}^{p}}{L_{m}^{q}}, \quad m^{*}=\frac{L_{m}^{I}}{L_{m}^{q}}
$$

with the density and $\beta$ dependence of $L_{m}^{q}$ leading to nonlinear behavior of $\Sigma_{v}$ and $m^{*}$ (even if we assume $W_{m}^{2}=W_{0}^{2}$ ). The nonlinear dependence of rhs of Eq. (112) on $W_{m}^{2}$ also cause the nonlinear contributions to $\Sigma_{v}$ and $m^{*}$. However, they are numerically less important.

Now we include the terms $\ell^{j}, u^{j}$ and $\omega^{j}$ in succession in lhs of Eqs. (57)-(159).

\section{The role of the lowest order local condensates}

As we have seen, these are the contributions, which contain the vector condensate $v(\rho)$, gluon condensate $g(\rho)$ and the scalar condensates $\kappa_{m}$ and $\zeta_{m}$ - Eqs. (11), (13), (14), (18). Account of these terms corresponds to the one-meson exchanges between the nucleon under consideration and the nucleon of the matter, with the point-like structures of the mesonnucleon vertices, Fig. 2(a). The solution can be obtained by using Eqs. (68)-(70) for the functions $\ell_{j}$. As we have seen, there is a simple solution, expressed by Eqs. (73) -(75)). The procedure of minimization of the difference between the lhs and rhs of Eqs. (57)-(59) indeed prefers the values $W_{m}(\rho, \beta) \approx W_{m}(\rho, 0)$. Thus Eqs. (173) and (74) appear to be true with good accuracy. Hence,

$$
\begin{aligned}
& \Sigma_{v}^{(p)}(\rho, \beta)=\Sigma_{v}(\rho, 0)\left(1-\frac{\beta}{4}\right), \\
& \Sigma_{v}^{(n)}(\rho, \beta)=\Sigma_{v}(\rho, 0)\left(1+\frac{\beta}{4}\right), \\
& m^{*(p)}\left(\rho, \beta, \kappa_{N}, \zeta_{N}\right)=m^{*}\left(\rho, 0, \kappa_{N}+\beta \zeta_{N}, 0\right), \\
& m^{*(n)}\left(\rho, \beta, \kappa_{N}, \zeta_{N}\right)=m^{*}\left(\rho, 0, \kappa_{N}-\beta \zeta_{N}, 0\right) .
\end{aligned}
$$

Thus, in the matter with the excess of the neutrons $(\beta>0)$, we obtain $\Sigma_{v}^{(n)}>\Sigma_{v}^{(p)}$, and $m^{*(n)}>m^{*(p)}$. For example, using the value $\Sigma_{v}(\rho, 0)$ obtained by sum rules approach in 
[16] $\left(\Sigma_{v}(\rho, 0)=335 \mathrm{MeV}\right)$, we find $\Sigma_{v}^{(n)}-\Sigma_{v}^{(p)}=170 \mathrm{MeV}, m^{*(n)}-m^{*(p)}=50 \mathrm{MeV}$ for the neutron matter $(\beta=1)$ at $\rho=\rho_{0}$.

The minimization procedure choses $W_{m}^{2}(\beta=-1)=2.50 \mathrm{GeV}^{2}, W_{m}^{2}(\beta=0)=2.30 \mathrm{GeV}^{2}$, $W_{m}^{2}(\beta=1)=2.05 \mathrm{GeV}^{2}, \Sigma_{v}^{(n)}-\Sigma_{v}^{(p)}=170 \mathrm{MeV}$ and $m^{*(n)}-m^{*(p)}=50 \mathrm{MeV}$. Thus, Eqs. (113) work well indeed. However, this approximation is not sufficient for the description of the potential energy $U(\rho, \beta)(24)$, providing $U>0$ for both symmetric and asymmetric cases.

\section{The role of the four-quark condensates}

Now we include the four-quark condensate, i.e., we use Eq. (60) for $L^{j}=\ell^{j}+\omega^{j}$, with $\omega^{j}$ described by Eqs. (110). Inclusion of these terms mimics several contributions on the rhs of the sum rules (157)-(58). In the condensates $\bar{u} \Gamma^{X} u \bar{u} \Gamma^{X} u$, presented by Eq. (103) the first term, which has a nonvanishing value only if $\Gamma^{X}=I$, generates a contribution to the $\hat{q}$ structure due to the anomalous Lorentz structure of the interaction between the scalar field and the nucleon, caused by the chiral-odd vacuum condensate $\langle 0|\bar{q} q| 0\rangle$. In similar way the first term on the rhs of Eq. (104) describes the contribution of the vector meson exchange to the scalar structure of the nucleon propagator. The anomalous Lorentz structures emerge if the nucleon-meson vertices are treated beyond the lowest order. These contributions are illustrated by Fig. 2(b). The second terms on the rhs of Eqs. (103) and (104) and on the rhs of Eq. (105) describe exchanges by the four-quark strongly correlated system (see Fig. 2(c)). The condensates presented by Eq. (105) have the same values for the proton and neutron. Thus their contributions do not depend on $\beta$.

The differences between the neutron and proton characteristics in the neutron matter at $\rho=\rho_{0}$ become $\Sigma_{v}^{(n)}-\Sigma_{v}^{(p)}=140 \mathrm{MeV}, m^{*(n)}-m^{*(p)}=-110 \mathrm{MeV}$. In the simplified model for the pion with the current masses of the constituent quarks, where $A_{4 q}^{q}(\beta)$ is given by Eq. (109), we obtain $\Sigma_{v}^{(n)}-\Sigma_{v}^{(p)}=145 \mathrm{MeV}$, while $m^{*(n)}-m^{(p)}=-110 \mathrm{MeV}$ at these values of $\rho$ and $\beta$.

\section{The final results}

These contributions come from the account of the $x$-dependence of the expectation values of the vector operators $\left\langle M\left|\bar{q}^{i}(0) \gamma_{\mu} q^{i}(x)\right| M\right\rangle$ with $q^{i}(x)$ defined by Eq. (63). As we have seen, the nonlocality of the scalar condensates is not important in our case. The nonlocality is included by putting $L^{j}=\ell^{j}+\omega^{j}+u^{j}$ with $u^{j}$ defined by Eq. (85). We use the structure functions, obtained in [30] for the calculation of the terms $u^{q}$ and $u^{p}$.

Account of the nonlocality of the vector condensate corresponds to inclusion of the formfactor of the vertex of the interaction between the vector meson and the nucleon of the matter - Fig. 2(d). Recall that similar contribution for the effective scalar meson exchanges vanishes in our approximation - Sec. III.B. 
We find the dependence of the nucleon vector self-energy $\Sigma_{v}$ and of the effective mass $m^{*}$ on density of the matter and on the asymmetry parameter $\beta$ and show the results in Figs. 3 and 4. For example, the differences between the neutron and proton characteristics in the neutron matter at $\rho=\rho_{0}$ are $\Sigma_{v}^{(n)}-\Sigma_{v}^{(p)}=110 \mathrm{MeV}, m^{*(n)}-m^{*(p)}=-70 \mathrm{MeV}$. In the simplified model for the pion with the current quark masses, where $A_{4 q}^{q}(\beta)$ is given by Eq. (109) we obtain $\Sigma_{v}^{(n)}-\Sigma_{v}^{(p)}=115 \mathrm{MeV}, m^{*(n)}-m^{*(p)}=-65 \mathrm{MeV}$ at these values of $\rho$ and $\beta$. The minor change is due to the small change in the $\beta$ dependence of the condensate $A_{4 q}^{q}$.

Due to the nonlinear character of Eqs. (57)-(59) the self-energies $\Sigma_{v}(\rho, \beta)$ and $\Sigma_{s}^{*}(\rho, \beta)=$ $m^{*}(\rho, \beta)-m$ could have been nonlinear in both $\rho$ and $\beta$. The nonlinear behavior of these characteristics with $\rho$ manifests itself explicitly. However, the dependence on $\beta$ appears to be linear in framework of the accuracy of our computations (see the next Subsection). Thus both $\Sigma_{v}$ and $\Sigma_{s}^{*}$ can be approximated by linear functions of $\beta$

$$
\begin{aligned}
& \Sigma_{v}(\rho, \beta)=\frac{\rho}{\rho_{0}}\left(V_{1}(\rho)+\beta \tau_{z} V_{2}(\rho)\right), \\
& \Sigma_{s}^{*}(\rho, \beta)=\frac{\rho}{\rho_{0}}\left(S_{1}(\rho)+\beta \tau_{z} S_{2}(\rho)\right)
\end{aligned}
$$

with $\tau_{z}=1$ for the proton, $\tau_{z}=-1$ for the neutron. The functions $V_{1,2}(\rho)$ and $S_{1,2}(\rho)$ are shown in Fig. 5. They can be approximated by polynomials of the second order - see Appendix D.

The single-particle potential energy is expressed by Eq. (24). At $\rho=\rho_{0}$ the neutronproton difference of the potential energy caused by the isovector interaction is $\Delta U_{n p} \approx$ $38 \beta \mathrm{MeV}$ at small $\beta$. In Fig. 6 we show the dependence $U(\rho)$ for several values of $\beta$ for both neutrons and protons. Recall that the potential energy is determined with the lower accuracy then the self-energies.

The nucleon residue $\lambda_{m}^{2}$ and the spectrum threshold $W_{m}^{2}$ exhibit very weak dependence on $\beta$. Thus we can assume

$$
\lambda_{m}^{2}(\rho, \beta) \approx \lambda_{m}^{2}(\rho, 0), \quad W_{m}^{2}(\rho, \beta)=W_{m}^{2}(\rho, 0) .
$$

\section{B. Explicit expression for the nucleon parameters in terms of QCD condensates}

We can present an approximate solution of Eqs. (57)-(59), in which the nucleon selfenergies are expressed in terms of the QCD condensates explicitly. We see from Eq. (115), that $W_{m}^{2}(\rho, \beta) \approx W_{m}^{2}(\rho, 0)$, while $W_{m}^{2}(\rho, 0)$ is close to it's vacuum value $W_{0}^{2}$ Eq. (46) (16]. Thus, we can put $W_{m}^{2}=W_{0}^{2}$ in the rhs of Eqs. (152)-(154) and (112). This enables us to present the proton characteristics $\Sigma_{v}$ and $m^{*}$ as the explicit functions of the quark condensates and of the Borel mass $M^{2}$ :

$$
\Sigma_{v}=-\frac{T_{v}^{p}\left(v_{N}+\frac{3}{4} \beta v_{N}^{(-)}\right)+T_{u 1}^{p}+\beta T_{u 2}^{p}+T_{\omega}^{p} A_{4 q}^{p}(\beta)}{1+F^{q}(\rho, \beta)} \frac{\rho}{\rho_{0}},
$$




$$
m^{*}=\left[m+\left(T_{\kappa}^{I}\left(\kappa_{N}+\beta \zeta_{N}\right)+T_{\omega}^{I} A_{4 q}^{I}(\beta)\right) \frac{\rho}{\rho_{0}}\right] \frac{1}{1+F^{q}(\rho, \beta)}
$$

with

$$
F^{q}(\rho, \beta)=\left[T_{v}^{q} v_{N}+m T_{g}^{q} g_{N}+T_{u 1}^{q}+\beta T_{u 2}^{q}+T_{\omega}^{q} A_{4 q}^{q}(\beta)\right] \frac{\rho}{\rho_{0}} .
$$

We denote $T_{\omega}^{i}=T_{\omega}^{i}\left(M^{2}\right)$ and $T_{j}^{i}=T_{j}^{i}\left(M^{2}, W_{0}^{2}\right)$ for other $j$ and introduce for $i=q, p, I$

$$
\begin{aligned}
& T_{k}^{i}\left(M^{2}, W_{0}^{2}\right)=\rho_{0} f_{k}^{i}\left(M^{2}, W_{0}^{2}\right) \frac{e^{m^{2} / M^{2}}}{\lambda_{0}^{2}}, \quad(k=v, g, \kappa), \\
& T_{u r}^{i}\left(M^{2}, W_{0}^{2}\right)=\rho_{0} u_{N, r}^{i}\left(M^{2}, W_{0}^{2}\right) \frac{e^{m^{2} / M^{2}}}{\lambda_{0}^{2}}, \quad(r=1,2), \\
& T_{\omega}^{i}\left(M^{2}\right)=\rho_{0} f_{4 q}^{i} \frac{e^{m^{2} / M^{2}}}{\lambda_{0}^{2}}
\end{aligned}
$$

with the functions $f_{k}^{q}$ and $f_{4 q}^{q}$ defined by Eqs. (172) and (110). It is instructive to present the density $\rho$ in units of the observable saturation density of the symmetric matter $\rho_{0}=$ $0.17 \mathrm{fm}^{-3}$.

Note that the functions $T_{j}^{i}\left(M^{2}\right)$ defined by Eq. (119) $(j=v, g, \kappa, u 1, u 2 ; i=q, p, I)$ depend on $M^{2}$ rather weakly. Thus, approximating

$$
T_{j}^{i}\left(M^{2}\right)=C_{j}^{i},
$$

we can replace the functions $T_{j}^{i}\left(M^{2}\right)$ in the lhs of Eqs. (119) by the constant coefficients $C_{j}^{i}$. Numerically most important functions $T_{v}^{p}\left(M^{2}\right)$ and $T_{\kappa}^{I}\left(M^{2}\right)$ can be approximated by the constant values with the errors of about $4 \%$ and $7 \%$. The largest errors of about $25 \%$ emerge in the averaging of the functions $T_{\omega}^{i}$. This solves the problem of expressing the in-medium change of nucleon parameters through the values of the condensates. For the proton

$$
\begin{gathered}
\Sigma_{v}=-\left(C_{v}^{p} v_{N}+\beta C_{v^{(-)}}^{p} v_{N}^{(-)}+m C_{u 1}^{p}+\beta m C_{u 2}^{p}+m C_{\omega}^{p} A_{4 q}^{p}(\beta)\right) \frac{\rho}{\rho_{0}} \frac{1}{\left(1-\mathcal{F}_{q}\right)}, \\
m^{*}=\left[m+\left(C_{\kappa}^{I} \kappa_{N}+\beta C_{\zeta}^{I} \zeta_{N}+C_{\omega}^{I} A_{4 q}^{I}(\beta)\right) \frac{\rho}{\rho_{0}}\right] \frac{1}{\left(1-\mathcal{F}_{q}\right)},
\end{gathered}
$$

with

$$
\mathcal{F}_{q}=-\left(C_{v}^{q} v_{N}+m C_{g}^{q} g_{N}+m C_{u 1}^{q}+\beta m C_{u 2}^{q}+m C_{\omega}^{q} A_{4 q}^{q}(\beta)\right) \frac{\rho}{\rho_{0}} .
$$

The coefficients in the rhs of Eqs. (121)-(123) are

$$
\begin{aligned}
& C_{v}^{q}=-0.062, \quad C_{g}^{q}=0.011 \mathrm{GeV}^{-1}, \quad C_{\omega}^{q}=-0.070 \\
& C_{u 1}^{q}=-0.074, \quad C_{u 2}^{q}=0.008, \\
& C_{\kappa}^{I}=-0.042 \mathrm{GeV}, C_{\zeta}^{I}=-0.042 \mathrm{GeV}, C_{\omega}^{I}=-0.063 \mathrm{GeV}, \\
& C_{v}^{p}=-0.090 \mathrm{GeV}, C_{v}^{p}=-0.068 \mathrm{GeV}, C_{\omega}^{p}=-0.095 \\
& C_{u 1}^{p}=0.094, \quad C_{u 2}^{p}=-0.020 .
\end{aligned}
$$


Note that the dependence of $\mathcal{F}_{q}$ on $\beta$ is very weak. (Recall that the lowest order OPE terms in the $\hat{q}$ structure of polarization operator do not depend on $\beta$.) Thus, in the lhs of Eqs. (121), (122) only the dependence of the numerators on $\beta$ is important. This explains the linear dependence of the self-energies $\Sigma_{v}$ and $\Sigma_{s}^{*}$ on $\beta$.

The values of $\Sigma_{v}, m^{*}$ and $\mathcal{F}_{q}$ for the neutron are described by Eqs. (121)-(123) with $\beta$ changed to $-\beta$. Equations (121), (122) enable to obtain $\Sigma_{v}$ and $m^{*}$ during the successive inclusion of condensates of the higher dimensions. If only the leading OPE terms are included the values provided by Eqs. (121), (122) actually coincide with the solutions of Eqs. (57)(59). If all the contributions are included Eqs. (121), (122) reproduce the values of $\Sigma_{v}$ and $m^{*}$ with the accuracy of $15 \%$ and $10 \%$ correspondingly for the symmetric matter. The precision of Eqs. (121) and (122) changes with $\beta$. For the neutron matter these equations provide the values $\Sigma_{v}^{(n)}-\Sigma_{v}^{(p)}=120 \mathrm{MeV}$ and $m^{*(n)}-m^{*(p)}=-60 \mathrm{MeV}$, comparing to the values of $110 \mathrm{MeV}$ and $-70 \mathrm{MeV}$, obtained in the previous subsection from the general solution of Eqs. (57)-(59).

\section{DISCUSSION}

Now we compare our results to those, obtained by nuclear physics methods. The lowest order OPE terms in the lhs of the sum rules describe mainly the exchanges by the localized $\bar{q} q$ pairs. This corresponds to the vector and (effective) scalar meson exchanges between the nucleon and the nucleons of matter. These exchanges have the point-like vertices and the standard Lorentz structures.

Inclusion of the higher order OPE terms corresponds to a more complicated picture of the meson exchanges between the nucleons. Turning to the four-quark condensates, we can separate the two types of terms. In the "factorized" contributions one of the $\bar{q} q$ operators is averaged over vacuum. In the "internal" terms both $\bar{q} q$ pairs act inside the nucleons , Eqs. (91), (92), (103)-(105). The first (factorized) term in the rhs of Eq. (103) describes the contribution to the $q_{\mu} \gamma^{\mu}$ structures of the polarization operator, which contains the scalar expectation value $\langle N|\bar{u} u| N\rangle$. In similar way the first (factorized) term in the rhs of Eq. (104) contributes to the scalar structure of the polarization operator, being proportional to the vector expectation value $\left\langle N\left|\bar{u} \gamma_{\mu} u\right| N\right\rangle$. These terms correspond to the anomalous Lorentz structures of the nucleon-meson vertices. As to the "internal" terms, i.e., the last terms on the rhs of Eqs. (91), (92), they can be interpreted as the exchanges by two-meson systems with their local interactions with the nucleon or as the exchanges by four-quark mesons (if there are any).

Inclusion of the nonlocal vector condensates $\bar{q}(0) \gamma_{\mu} q(x)$ means that the vertices of the interactions between the nucleons of the matter and the vector mesons do not have a pointlike structure, requiring rather description by the formfactors. The nonlocality of the scalar condensate does not influence the results in our approach.

A usual subject of calculation is the difference between the characteristics of neutron and proton. If only the lowest OPE terms are included, the vector self-energies are determined by 
the vector condensates. The neutron-proton difference $\Sigma_{v}^{(n)}-\Sigma_{v}^{(p)}$, usually attributed to the $\rho$ meson exchange is $170 \mathrm{MeV}$ at $\rho=\rho_{0}$ and $\beta=1$. Inclusion of the four-quark condensates and of the nonlocalities subtract $30 \mathrm{MeV}$ and $28 \mathrm{MeV}$ from this value. The lowest order OPE terms provide the difference of the effective masses $m^{*(n)}-m^{*(p)}=50 \mathrm{MeV}$ at the same values of $\rho$ and $\beta$. Inclusion of the four-quark condensates and of the nonlocalities adds $(-160 \mathrm{MeV})$ and $40 \mathrm{MeV}$. This leads to $\Sigma_{v}^{(n)}-\Sigma_{v}^{(p)}=110 \mathrm{MeV}$ and $m^{*(n)}-m^{*(p)}=$ $-70 \mathrm{MeV}$ in neutron matter at $\rho=\rho_{0}$

The structure of the equations for $\Sigma_{v}$ amd $m^{*}$ (121), (122) is similar to that (23) employed in nuclear physics. Recall that in the Hartree approximation dependence of $\Sigma_{v}$ on the density deviates from the linear law due to $\Sigma_{q}(23)$. (The same refers to $\Sigma_{s}^{*}$ if the nucleon Fermi motion is neglected). In our approach the nonlinear behavior of the self energies is due to nonzero values of $\mathcal{F}_{q}$.

Now we compare the numerical results. Considering the papers, containing relativistic calculations, we can compare the vector and scalar self-energies $\Sigma_{v}$ and $\Sigma_{s}^{*}=m^{*}-m$. In the case of the works, carried out in the nonrelativistic approximation, we can compare the nucleon potential energies $U^{n, p}$. We analyze also the contribution to the parameter, conventionally denoted as $a_{4}$ 31, which is defined as

$$
\varepsilon\left(\rho_{0}, \beta\right)=\varepsilon\left(\rho_{0}, 0\right)+\beta^{2} a_{4}+O\left(\beta^{4}\right),
$$

being thus the lowest order term of $\beta^{2}$ expansion of the averaged binding energy $\varepsilon$ per nucleon at saturation value of density.

Of course, we cannot expect very good agreement, since our calculations are carried out in the gas approximation. The future and more sophisticated calculations should include the scalar and four-quark condensates beyond the gas approximation. This would correspond to the account of the renormalization of the nucleon interactions with the matter by the particle-hole excitations in rhs of the sum rules. Another reason is that the results should be corrected for the effects of antisymmetrization of the total final state wave function ("exclusion effect") 32 .

The general feature of the relativistic calculations is that they provide the positive value of the difference $\Sigma_{v}^{(n)}-\Sigma_{v}^{(p)}>0$ in the matter with the neutron excess. Also the proton effective masses are above the neutron ones in this case, i.e., $m^{*(n)}-m^{*(p)}<0$. Our calculations show the same tendency. As to the quantitative results, our values of $\Sigma_{v}^{(n)}-\Sigma_{v}^{(p)}$ and $m^{*(n)}-m^{*(p)}$ appear to be about twice smaller than those, obtained in [33]. Our value of the effective mass splitting is also about two times smaller than the result of [34 but is only $30 \%$ smaller than that of [35]. However, we find somewhat smaller discrepancy with the relativistic Brueckner-Hartree-Fock (RBHF) calculations, presented in [36]. They found $\Sigma_{v}^{(n)}-\Sigma_{v}^{(p)} \approx 30 \mathrm{MeV}$ and $m^{*(n)}-m^{*(p)} \approx-25 \mathrm{MeV}$ at $\beta=0.2$ (Fig. 3 of [36]), while our results are $\Sigma_{v}^{(n)}-\Sigma_{v}^{(p)} \approx 20 \mathrm{MeV}, m^{*(n)}-m^{*(p)} \approx-15 \mathrm{MeV}$. Another RBHF analysis [37] provided the results, which are very close to our ones. One can extract the values $\Sigma_{v}^{(n)}-\Sigma_{v}^{(p)} \approx 80 \mathrm{MeV}$, and $m^{*}(n)-m^{*}(p) \approx-50 \mathrm{MeV}$ at $\beta=0.75$ from Figs. 10 and 11 of [37. Our values are $80 \mathrm{MeV}$ and $55 \mathrm{MeV}$ correspondingly. Note, however, that the split of the effective masses, obtained in [37] is due to the exchange effects only. 
The nonrelativistic calculations, carried out in various approaches [38, 39] provide $U^{(n)}-$ $U^{(p)} \approx 60 \mathrm{MeV}$ at $\rho=\rho_{0}$ and $\beta=1$. This is consistent with the earlier calculations [40]. Our value is $40 \mathrm{MeV}$ at $\rho=\rho_{0}$ and $\beta=1$.

Another important parameter is the symmetry energy - Eq. (125). Note that we calculate the quantity

$$
\Delta \varepsilon=\frac{1}{2 \rho}\left(U^{(n)} \rho_{n}+U^{(p)} \rho_{p}\right)
$$

which is the true contribution to the energy per nucleon. Using Eq. (114) we can express $\Delta \varepsilon=\frac{1}{2}\left(V_{1}+S_{1}-\beta^{2}\left(V_{2}+S_{2}\right)\right)$. The value

$$
\Delta \varepsilon\left(\rho_{0}, \beta\right)-\Delta \varepsilon\left(\rho_{0}, 0\right)=\beta^{2} \Delta a_{4}+O\left(\beta^{4}\right),
$$

thus being the true contribution of the isovector forces. Our value is $\Delta a_{4} \approx 10 \mathrm{MeV}$. This is close to the one, obtained in 32 . The $\beta^{2}$ law is true up to $\beta^{2}=1$ with $10 \%$ accuracy in agreement with [36, 38, 39]. To find the total contribution of the potential energy one must include the term caused by the exclusion effect, mentioned above. This adds $9 \mathrm{MeV}$ to $a_{4}$ 32. Including also the contribution of the kinetic energy, we obtain $a_{4}=29 \mathrm{MeV}$. The various calculations of this parameter provide the values around $30 \mathrm{MeV}$ [33]-[41]. Thus, our result agrees with those, obtained by nuclear physics methods.

\section{SUMMARY}

We expressed the vector and scalar self-energies of a nucleon in asymmetric nuclear matter as function of density $\rho$ and of the asymmetry parameter $\beta$. We presented the nucleon characteristics in terms of the in-medium expectation values of QCD operators. The main ingredients are the nonlocal vector condensates $\left\langle M\left|\bar{u}(x) \gamma_{0} u(0) \pm \bar{d}(x) \gamma_{0} d(0)\right| M\right\rangle$, the scalar condensates $\langle M|\bar{u}(0) u(0) \pm \bar{d}(0) d(0)| M\rangle$ and the four-quark condensates. The local vector condensates are calculated easily. The nonlocality of the vector condensates is expressed in terms of the nucleon structure functions. The scalar condensate $\kappa(\rho)=$ $\langle M|\bar{u} u+\bar{d} d| M\rangle$ is presented in terms of the observable sigma term. The scalar condensate $\zeta(\rho, \beta)=\langle M|\bar{u} u-\bar{d} d| M\rangle$ and the four-quark condensates are calculated in framework of perturbative chiral quark model (PCQM).

Although we treat the condensates in the gas approximation, the nucleon characteristics are not linear in density. The corresponding equations (121), (122) are analogous to the equations of nuclear physics beyond the mean field approximation. Also Eqs. (121), (122) provide explicit expression of the nucleon characteristics in terms of the QCD condensates.

The successive inclusion of the OPE terms in the lhs of the sum rules finds direct analogs in the meson-exchange description of the interactions of the nucleon in nuclear matter. The lowest order OPE terms correspond to the exchanges by the vector and (effective) scalar mesons with the point-like vertices of the interactions. The higher order terms correspond to the nonlocal structure of nucleon-meson vertices, including the anomalous Lorentz structures,

and to the exchanges by the strongly correlated four-quark systems. A possible interpretation 
of the latter contributions is a local two-meson exchange (or the exchanges by the four-quark mesons, if there are any [42]), Figs. 1, 2.

We obtained the functions $\Sigma_{v}(\rho, \beta)$ and $m^{*}(\rho, \beta)$ for all values of $\beta$. We calculated also the single-particle potential energy $U(\rho, \beta)$. The results are presented in Figs. 3-6.

Note, that we did not need phenomenological parameters of the nucleon-meson interactions. We used the condensates which have been either calculated, or expressed in terms of the observables. For the four-quark condensates we used, however, the already known input parameters of PCQM. While including the condensates of the higher dimension in succession, we found the direct analogs of the meson-nucleon exchange mechanisms of nuclear physics.

Our results for the nucleon self-energies are in reasonable agreement with the results of nuclear physics. The value of the symmetry energy is close to the one obtained by the nuclear physics methods.

The work was supported by the grants RFBR - 03-02-17724 and RSGSS - 1124.2003.2.

\section{APPENDIX A}

Here we discuss the isotopic structure of the nucleon current (28).

The current (28) does not have a definite isospin. This is not important for the calculations in a medium with the isospin $\mathrm{I}=0$, e.g., in vacuum or in symmetric nuclear matter. In these cases the expectation values of $\bar{u} u$ and $\bar{d} d$ operators have equal values (the same is true for more complicated condensates). This reasoning does not work for asymmetric matter.

Therefore it is reasonable to start with the pure $I=1 / 2$ current

$$
\begin{gathered}
j(y)=\epsilon_{a b c} \cdot\left[\sqrt{\frac{2}{3}} u^{a}(y) C \gamma_{\mu} u^{b}(y) \gamma_{5} \gamma^{\mu} d^{c}(y)\right. \\
\left.-\frac{1}{\sqrt{6}} u^{a}(y) C \gamma_{\mu} d^{b}(y) \gamma_{5} \gamma^{\mu} u^{c}(y)-\frac{1}{\sqrt{6}} d^{a}(y) C \gamma_{\mu} u^{b}(y) \gamma_{5} \gamma^{\mu} u^{c}(y)\right]
\end{gathered}
$$

However it turns out that even in asymmetric matter an explicit calculation of the polarization operator by using the current (A1) provides the same result as that with the original Ioffe current (28).

This is due to the Fermi statistics of the quarks. In the OPE the current (28) annihilates a local three-quark state. The coordinate function of the three quarks is symmetric. The color structure is antisymmetric as well as the spin structure of the spin three quark system (clearly the current (28) has spin 1/2). Thus we need the antisymmetric isospin wave function. Due to the Fermi statistic of the fermions any symmetric isospin component will vanish after the account for all possible permutations of the quarks. This means that only the isospin 1/2 component actually survives in the OPE expansion of the polarization operator (27) corresponding to the of current (28). In other words there is a relation between the contributions of the first and two last terms of the rhs of Eq. (A1). This provides a possibility to use (28) for the calculations in isospin asymmetric matter. 


\section{APPENDIX B}

The integrals in the rhs of Eq. (84) can be evaluated by using the formula

$$
\int \frac{d^{4} x}{x^{8}} x_{\mu} x_{\nu} e^{i\left(q^{\prime} x\right)}=\frac{1}{6}\left[g_{\mu \nu}+\frac{2 g_{\mu}^{\prime} q_{\nu}^{\prime}}{q^{\prime 2}}\right] \int \frac{d^{4} x}{x^{6}} e^{i\left(q^{\prime} x\right)}
$$

with $q^{\prime}=q-p \alpha$. The last factor is

$$
\int \frac{d^{4} x}{x^{6}} e^{i\left(q^{\prime} x\right)}=-\frac{i \pi^{2}}{8} q^{\prime 2} \ln \left(-q^{\prime 2}\right)+\ldots
$$

Here the dots denote the terms which will be killed by the Borel transform.

Thus the lhs of the sum rules contains the contribution of the form

$$
X=\int d \alpha \ln \left(Q^{2}+A^{2}(\alpha)\right) f(\alpha)
$$

with $Q^{2}=-q^{2}, A(\alpha)=\alpha \frac{\left(s-m^{2}\right)-m^{2} \alpha}{1+\alpha}$. We can present

$$
X=\ln Q^{2} \int_{0}^{1} d \alpha f(\alpha)+\int_{0}^{1} d \alpha \ln \frac{Q^{2}+A^{2}(\alpha)}{Q^{2}} f(\alpha) .
$$

The first term on the rhs contains the standard logarithmic factor with the cut, running to infinity. It is described by the "pole + continuum" model in a standard way. The second term contains a finite cut. It describes the singularities in the $u$-channel, caused by the nonlocal structure of the vector condensate, corresponding to the exchange terms on the rhs of the sum rules. We neglect such contributions, thus coming to the Hartree description of the nucleon in nuclear matter.

\section{APPENDIX C}

Here we calculate the contribution of the four-quark condensates to $I$ structure of the polarization operator $\Pi_{m}(q)$. The contribution is provided by the scalar-vector condensate $r_{N}^{S V}$ defined by Eq. (92) with $X=S, Y=V$. The first two terms determine the "factorized" contribution, which can be written as

$$
\Pi_{f}^{I}=-\frac{2}{3} \int \frac{d^{4}}{\pi^{2} x^{4}}\left(x, \theta^{u}(x)\right) e^{i(q x)}\langle 0|\bar{d} d| 0\rangle,
$$

with the vector $\theta_{\mu}^{u}(x)$ defined by Eq. (64). Neglecting for a while the nonlocal contributions, we put $\theta^{u}(x)=\theta^{u}(0)$. In this approximation

$$
\Pi_{f}^{I}=\Pi_{f, l o c}^{I}=-\frac{4}{3} \frac{(p q)}{q^{2} m}\langle 0|\bar{d} d| 0\rangle\left(2 \rho_{p}+\rho_{n}\right),
$$


thus corresponding to Eq. (102) with $a_{p}^{S V}=\frac{4}{3}\langle 0|\bar{d} d| 0\rangle, a_{n}^{S V}=\frac{2}{3}\langle 0|\bar{d} d| 0\rangle$. The "internal" contributions, provided by the second terms in the rhs of Eqs. (92) and (104) are also proportional to $(p q)$, with the coefficients $\left(a_{i}^{S V}\right)_{\text {int }}$ determined in [16]. The factorized local terms and the internal terms provide the rhs of the last equality of Eq. (102).

Taking into account the $x$-dependence of the vector $\theta_{\mu}^{u}(x)$ in Eq. (C1) we obtain a more accurate expression for the "factorized" contribution, which contains the second moments of the structure functions and the terms of the $x^{2}$ expansion of the integrand

$$
\begin{gathered}
\Pi_{d}^{I}=\frac{\langle 0|\bar{d} d| 0\rangle}{q^{2}} \frac{m}{3}\left[-4 \frac{(p q)}{m^{2}}\left(\left\langle\eta_{u}\right\rangle \rho_{p}+\left\langle\eta_{d}\right\rangle \rho_{n}\right)+2\left(\left\langle\alpha \eta_{u}\right\rangle \rho_{p}+\left\langle\alpha \eta_{d}\right\rangle \rho_{n}\right)\right. \\
\left.+\left(\left\langle\xi_{u}\right\rangle \rho_{p}+\left\langle\xi_{d}\right\rangle \rho_{n}\right)+\frac{1}{2} \frac{\mu_{0}^{2}}{m^{2}} \frac{1}{q^{2}}\left(\left\langle\eta_{u}\right\rangle \rho_{n}+\left\langle\eta_{d}\right\rangle \rho_{p}\right)\right] .
\end{gathered}
$$

The notations are explained in Eqs. (79) and (81), $\mu_{0}^{2}=0.8 \mathrm{GeV}^{2}$ (41), (42).

\section{APPENDIX D}

The functions $V_{1,2}(\rho)$ and $S_{1,2}(\rho)$ introduced by Eq. (114) can be approximated by the polynomials of the second order:

$$
P_{2}(x)=b_{0}+b_{1} x+b_{2} x^{2}
$$

with $x=\rho / \rho_{0}$. The values of the coefficients $b_{i}$ are presented in Table 1 . This leads to parametrization of the proton potential energy

$$
U(x)=\left[x\left(-0.15+0.17 x-0.09 x^{2}\right)+\beta x\left(-0.04 x+0.02 x^{2}\right)\right] \mathrm{GeV} .
$$

Table The values of the coefficients $b_{i}(\mathrm{GeV})$ of the polynomials $P_{2}(\mathrm{D} 1)$, which approximate the nucleon self-energies.

\begin{tabular}{|l|r|r|r|}
\hline & $b_{0}$ & $b_{1}$ & $b_{2}$ \\
\hline$V_{1}$ & 0.102 & 0.015 & 0.026 \\
\hline$V_{2}$ & -0.036 & -0.008 & -0.011 \\
\hline$S_{1}$ & -0.254 & 0.150 & -0.114 \\
\hline$S_{2}$ & 0.034 & -0.035 & 0.034 \\
\hline
\end{tabular}




\section{References}

[1] M.A. Shifman, A.I. Vainshtein and V.I. Zakharov, Nucl. Phys. B 147, 385, 448, 519 (1979).

[2] B.L. Ioffe, Nucl. Phys. B 188, 317 (1981).

[3] B.L. Ioffe and A.V. Smilga, Nucl. Phys. B 232, 109 (1984).

[4] M.A. Shifman, "Vacuum structure and QCD sum rules" (North Holland, Amsterdam, 1992).

[5] L.J. Reinders, H. Rubinstein, S. Yazaki, Phys. Rep. 127, 1 (1985).

[6] K.G. Wilson, Phys. Rev. 179, 1499 (1969).

[7] E.G. Drukarev and E.M. Levin, JETP Lett. 48, 338 (1988); Sov. Phys. JETP 68, 680 (1989).

[8] E.G. Drukarev and E.M. Levin, Nucl. Phys. A 511, 679 (1990); Prog. Part. Nucl. Phys. 27, 77 (1991).

[9] E.G. Drukarev and M.G. Ryskin, Nucl. Phys. A 578, 333 (1994).

[10] E.G. Drukarev, M.G. Ryskin and V.A. Sadovnikova, Prog. Part. Nucl. Phys. 47, 73 (2001).

[11] J. Gasser, Ann. Phys. 136, 62 (1981).

[12] U. Weidner et al., Phys. Rev. Lett. 58, 648 (1987).

[13] Ulf-G. Meisner, G. Smith et al., hep-ph/0011277.

[14] M.A. Shifman, A.I. Vainshtein and V.I. Zakharov, Phys. Lett. B 178, 443 (1978).

[15] X. Jin, T.D. Cohen, R.J. Furnstahl, and D.K. Griegel, Phys. Rev. C 49, 464 (1994).

[16] E.G. Drukarev, M.G. Ryskin, V.A. Sadovnikova, Th. Gutsche and Amand Faessler, Phys. Rev. C (in press).

[17] E.G. Drukarev, M.G. Ryskin, V.A. Sadovnikova, V.E. Lyubovitskij, Th. Gutsche and A. Faessler, Phys. Rev. D 68, 054021 (2003).

[18] T. Gutsche and D. Robson, Phys. Lett. B 229, 333 (1989).

[19] V.E. Lyubovitskij, Th. Gutsche and A. Faessler, Phys. Rev. C 64, 065203 (2001).

[20] V.E. Lyubovitskij, Th. Gutsche, A. Faessler and E.G. Drukarev, Phys. Rev. D 63, 054026 (2001). 
[21] V.E. Lyubovitskij, Th. Gutsche, A. Faessler and R. Vihn Mau, Phys. Rev. C 65, 025202 (2002);

V.E. Lyubovitskij, P. Wang, Th. Gutsche and A. Faessler, Phys. Rev. C 66, 055204 (2002).

[22] L.S. Celenza and C.M. Shakin, "Relativistic Nuclear Physics" (World Scientific, Philadelphia, 1986).

[23] F.J. Yndurain, "Quantum Chromodynamics", (Springer Verlag. NY, 1983).

[24] C. Itzykson and J.-B.Zuber, "Quantum Field Theory", (McGraw Hill, NY, 1980).

[25] V.M. Braun and A.V. Kolesnichenko, Nucl. Phys. B 283, 723 (1987).

[26] X. Jin, T.D. Cohen, R.J. Furnstahl, and D.K. Griegel, Phys. Rev. C 47, 2882 (1993).

[27] E.G. Drukarev, M.G. Ryskin, V.A. Sadovnikova and A. Faessler, Phys. Rev. C 65, 074051 (2002); E: 66039903 (2002).

[28] R.D. Carlitz and D.V. Creamer, Ann. Phys. 118, 429 (1979);

D.I. Diakonov and V.Yu. Petrov, Nucl. Phys. B 272, 457 (1986).

[29] J. Gasser, H. Leutwyler and M.E. Sainio, Phys. Lett. B 253, 252, 260 (1991).

[30] A.D. Martin, R.G. Roberts and W.J. Stirling, Phys. Lett. B 387, 419 (1996).

[31] A.L. Fetter and J.D. Walecka, "Quantum theory of many-particle systems" (McGrawHill, New-York, 1971).

[32] J.V. Noble, Nucl. Phys, A 329, 354 (1979).

[33] M. Lopez-Quelle, S. Marcos, R. Niembro, A. Bouyssy and N.V. Giai, Nucl. Phys. A 483, 479 (1988).

[34] B. Liu, V. Greco, V. Baran, M. Colonna and M. Di Toro, Phys. Rev. C 65, 045201 (2002).

[35] V. Greco, M. Colonna, M. Di Toro, G. Fabbri and F. Matera, Phys. Rev. C 64, 045203 (2001).

[36] F. de Jong and H. Lenske, Phys. Rev. C 57, 3099 (1998).

[37] H. Huber, F. Weber and M.K. Weigel, Phys. Rev. C 51, 1790 (1995).

[38] I. Bombaci and U. Lombardo, Phys. Rev. C 44, 1892 (1991).

[39] W. Zuo, I. Bombaci, U. Lombardo, Phys. Rev. C 60, 024605 (1999).

[40] A. Bohr and B. Mottelson, "Nuclear Structure", v.1 (Benjamin, NY, 1969). 
[41] R. Malfliet, Prog. Part. Nucl. Phys. 21, 207 (1988).

[42] D. P. Roy, J.Phys. G 30, R113 (2004). 


\section{Figure captions}

Fig.1. Successive inclusion of the OPE terms. The helix lines denote the current (28). Solid lines denote the quarks. Thick lines show the matter. (a) The local vector and scalar condensates are included (contribution of the gluon condensate is not shown). (b) The factorized part of the four-quark condensates is included. One of the $\bar{q} q$ operators acts on vacuum, while another $\bar{q} q$ operator acts inside the nucleon. Small circles stand for averaging over vacuum. (c) The internal parts of the four-quark operators are included. All the quark operators act inside the nucleon. (d) The nonlocality of the vector condensate is included. The dark blobs denote the nonlocal condensates.

Fig.2. The analogs of the OPE terms in the meson-exchange interaction of the nucleon with matter. Figures 2(a-d) correspond to the Figures 1(a-d). Solid line denotes the nucleon. Thick lines show the matter. Wavy and dashed lines stand for vector and scalar mesons. (a) Exchanges by vector and scalar ("effective") mesons with the pion vertices. (b) Exchanges by these mesons with the anomalous Lorentz structures of the vertices (the dark squares). (c) Exchanges by the $\bar{q} q$ pairs with strong correlations between them, presumably the local interactions with two mesons. Double line denotes the two-meson systems. (d) Inclusion of the nonlocal structure of the vertices of interaction between the vector mesons and the nucleons of the matter. The dark blobs denote the formfactors of the vertices.

Fig.3. The density dependence of the vector self-energy $\Sigma_{v}$ (solution of Eqs. (57)-(59)). The solid line shows the results for symmetric matter $(\beta=0)$. The dashed and dotted lines show the proton and neutron self-energies $\Sigma_{v}^{(p)}$ and $\Sigma_{v}^{(n)}$ in the neutron matter $(\beta=1)$.

Fig.4. The density dependence of the effective mass $m^{*}$ (solution of Eqs. (57)-(59)). The solid line shows the results for symmetric matter $(\beta=0)$. The dashed and dotted lines show the proton and neutron self-energies $m^{*(p)}$ and $m^{*(n)}$ in the neutron matter $(\beta=1)$.

Fig.5. The density dependence of the functions $x V_{1}, x V_{2}, x S_{1}$ and $x S_{2}$ introduced by Eqs. (114), $x=\frac{\rho}{\rho_{0}}$. The dotted lines demonstrate the quality of fitting with the simple functions on $\rho$, as described in Appendix D.

Fig.6. The density dependence of the single particle potential energy $U$, Eq. (24, The solid line shows the results for symmetric matter $(\beta=0)$. The dashed and dotted lines show the proton and neutron self-energies $U^{(p)}$ and $U^{(n)}$ in the neutron matter $(\beta=1)$. 

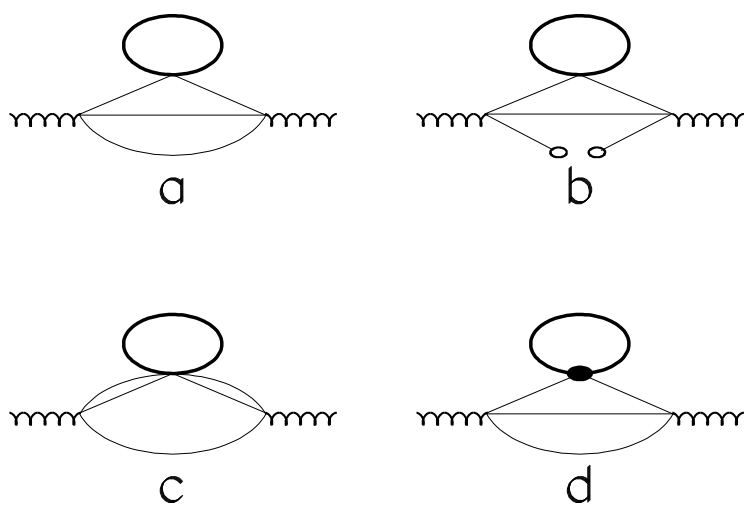

Figure 1:
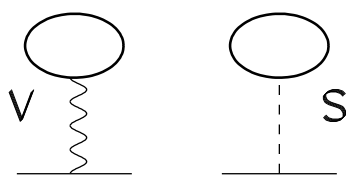

a

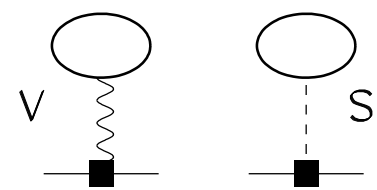

b
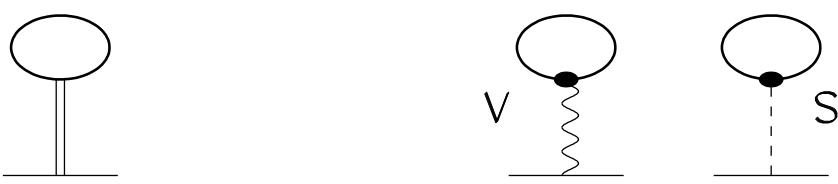

C

d

Figure 2: 


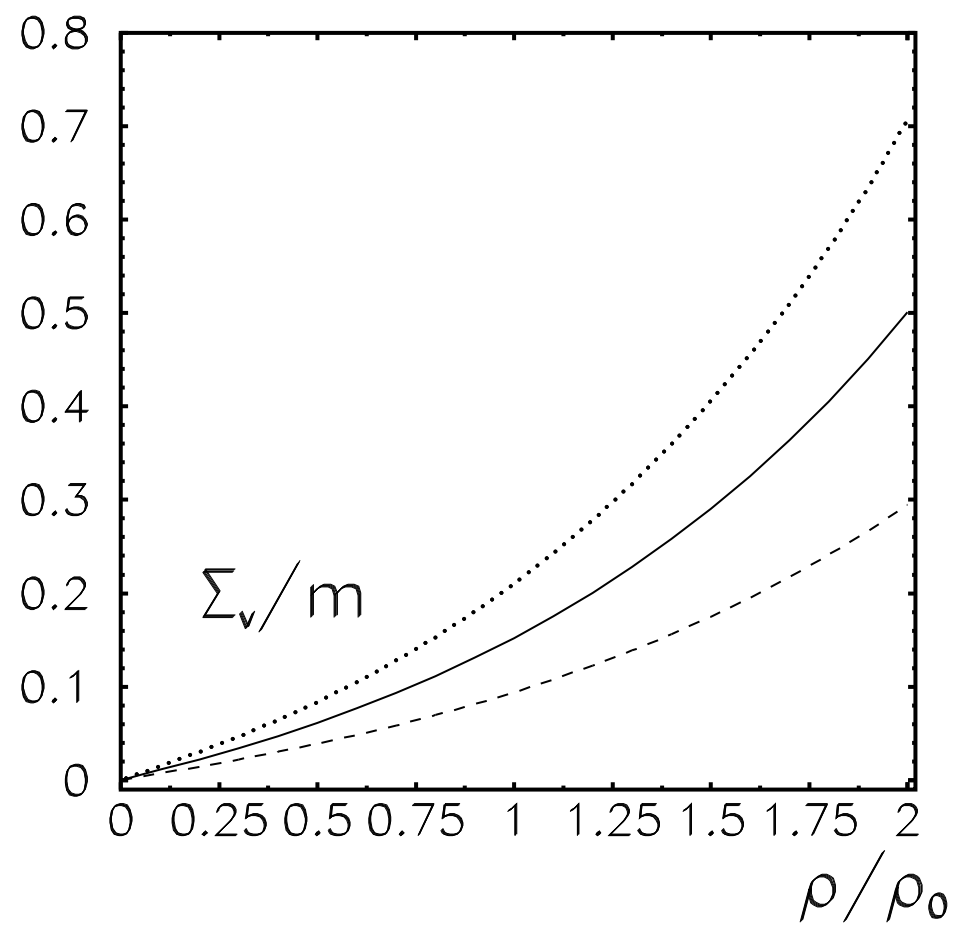

Figure 3: 


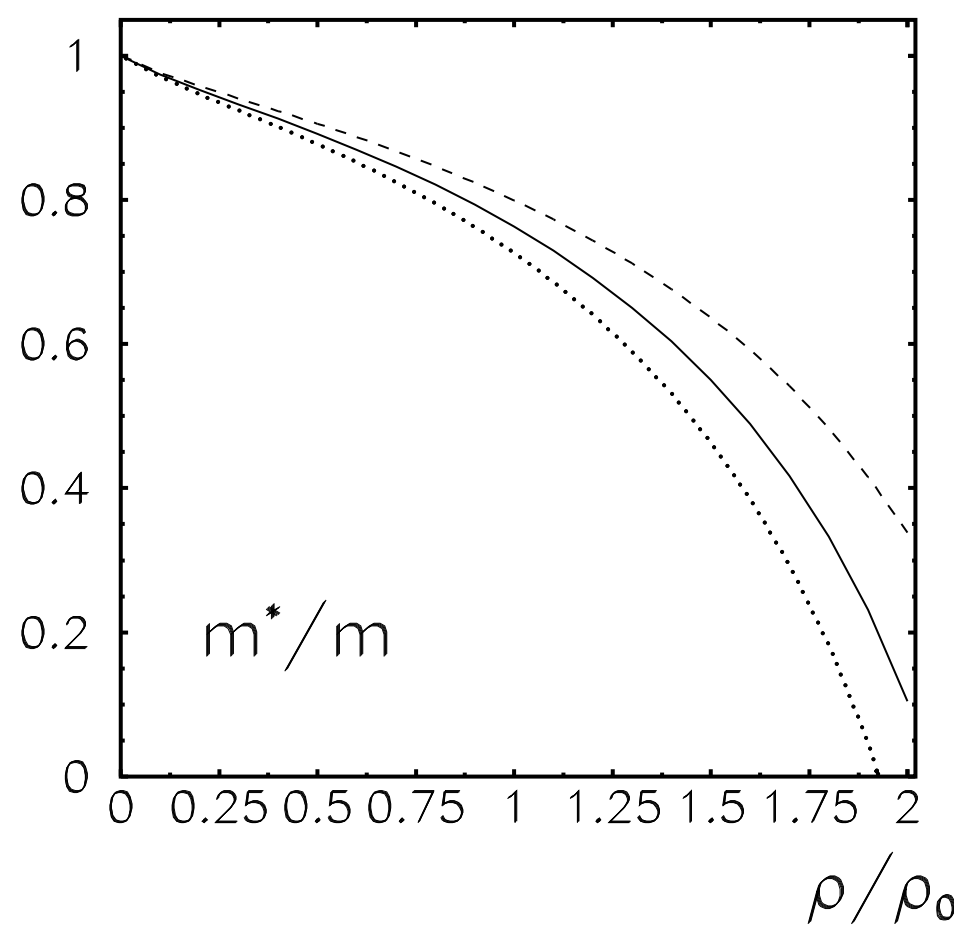

Figure 4: 


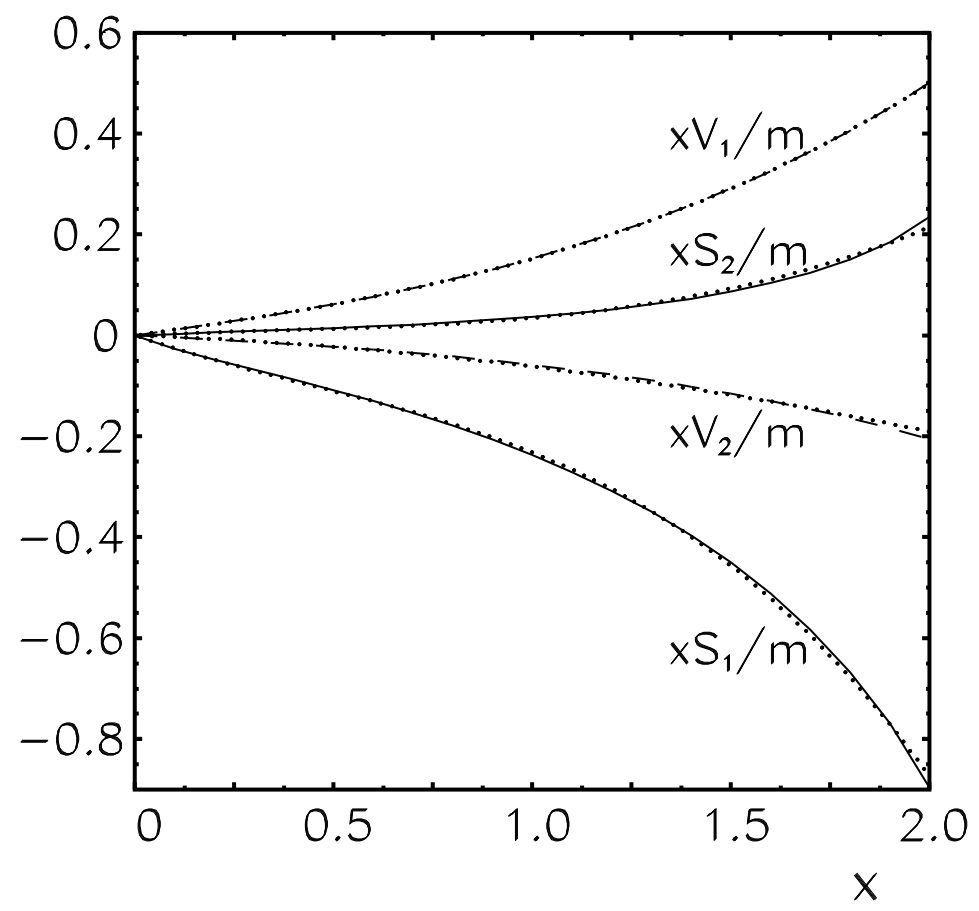

Figure 5: 


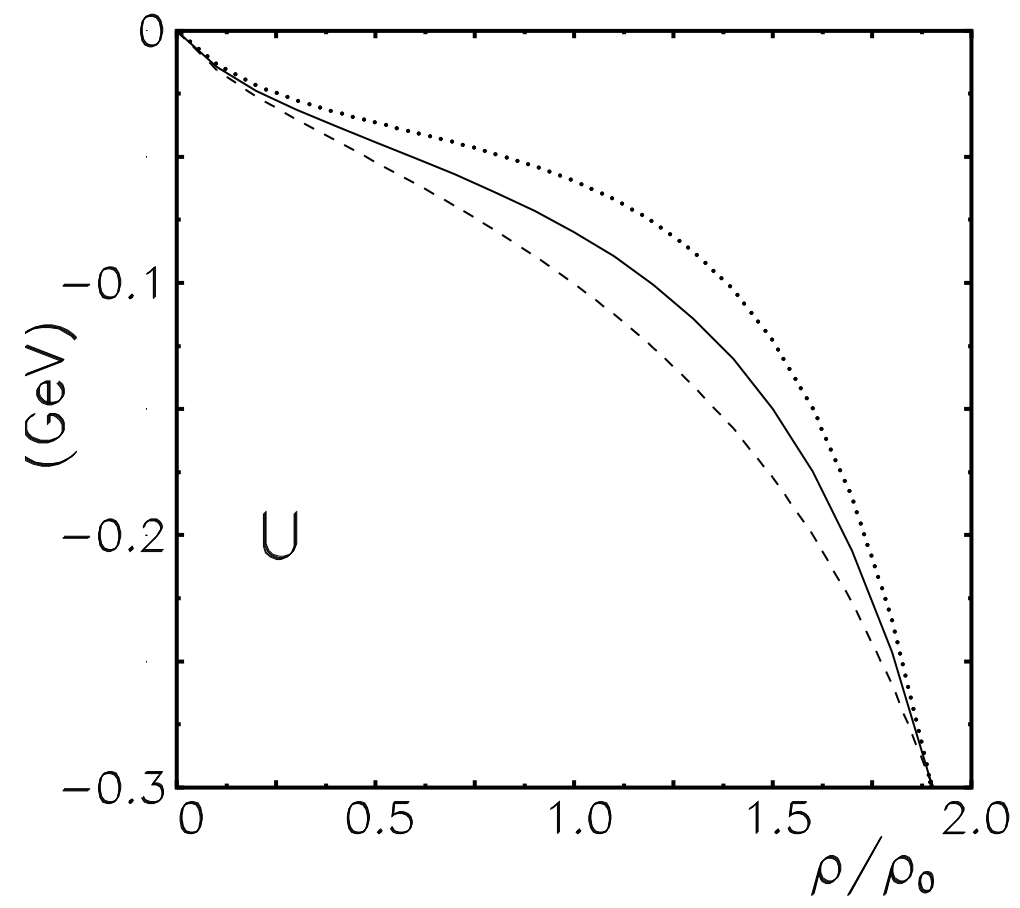

Figure 6: 